\title{
Optimal Liquidation under Stochastic Liquidity
}

\author{
Dirk Becherer*, Todor Bilarev ${ }^{\dagger}$, Peter Frentrup ${ }^{\ddagger}$ \\ Institute of Mathematics, Humboldt-Universität zu Berlin
}

November 3, 2017

\begin{abstract}
We solve explicitly a two-dimensional singular control problem of finite fuel type for infinite time horizon. The problem stems from the optimal liquidation of an asset position in a financial market with multiplicative and transient price impact. Liquidity is stochastic in that the volume effect process, which determines the inter-temporal resilience of the market in spirit of PSS11, is taken to be stochastic, being driven by own random noise. The optimal control is obtained as the local time of a diffusion process reflected at a non-constant free boundary. To solve the HJB variational inequality and prove optimality, we need a combination of probabilistic arguments and calculus of variations methods, involving Laplace transforms of inverse local times for diffusions reflected at elastic boundaries.

Keywords: Stochastic liquidity, transient price impact, optimal liquidation, stochastic volume effect, singular control, finite-fuel problem, free boundary, inverse local time, elastic reflection

MSC2010 subject classification: 35R35, 49J40, 49L20, 60H30, 60J50, 60J55, 93E20, 91G80
\end{abstract}

JEL classification: C02, C61, D99, G12, G33

\section{Introduction}

A typical stochastic optimal control problem in models of illiquid markets is a large trader (the controller) who optimizes her trading strategy such as to balance some trading objective against her adverse price impact, which causes (non-proportional)

\footnotetext{
*All authors would like to thank Wolfgang Runggaldier (Co-Editor) and the anonymous two referees and Associate Editor for very helpful comments.

${ }^{\dagger}$ T. Bilarev gratefully acknowledges support by German Science foundation DFG via Berlin Mathematical School BMS and research training group RTG1845 StoA.

${ }^{\ddagger}$ Email addresses: becherer,bilarev,frentrup@math.hu-berlin.de
} 
cost from illiquidity. In the majority of literature on price impact models the intertemporal impact is typically a deterministic function of the strategy of the (single) large trader. In reality, we would rather expect some aspects of market liquidity (where Kyl85 has distinguished resilience, depth and tightness) to vary stochastically over time, and a sophisticated trader to adapt her optimal strategy accordingly. Even for the extensively studied problem of optimal liquidation, there are relatively few recent articles on models in continuous time where the optimal liquidation strategy is adaptive to random changes in liquidity, cf. Alm12, LS13, FSU17, GHS16, GH16].

We consider a model where temporary market imbalances involve own stochasticity. Price impact is transient, i.e. it could be persistent but eventually vanishes over time. Moreover, it is non-linear, corresponds to a general shape for the density of the limit order book (see Remark 2.3), and is multiplicative to ensure positive risky asset prices. More precisely, our price process $S=\left(S_{t}\right)_{t \geq 0}=\left(f\left(Y_{t}\right) \bar{S}_{t}\right)_{t>0}$ observed in the market deviates by a factor $f\left(Y_{t}\right)$ from the fundamental price $\bar{S}_{t}$ that would prevail in the absence of large traders. The impact function $f$ is positive and increasing and thus the multiplicative structure ensures that prices stay positive, in contrast to the additive models where a conceptual drawback is that negative asset prices can occur with (small) positive probability. Our stochastic impact process $Y$ is of a controlled OrnsteinUhlenbeck (OU) type, namely it is driven by a Brownian motion and the large trader's holdings in the risky asset (see eq. (2.3) below). The mean-reversion of $Y$ models the transience of impact. Analogously to [AFS10, PSS11, the impact function $f$ can be linked to the shape of a limit order book (LOB) and $Y$ may be understood as a volume effect process describing the (temporal) imbalance in the LOB, see Remark 2.3. The additional noise in $Y$ gives a stochastic LOB, or it can be seen as the accumulated effect from other non-strategic large traders, see Remark 2.4.

For our multiplicative model with transient impact, we take the fundamental price $\bar{S}$ to be an exponential Brownian motion and permit for non-zero correlation with the stochastic volume effect process $Y$. In this setup, we study the optimal liquidation problem for infinite time horizon as a singular stochastic control problem of finite fuel type and construct its explicit solution. Our main result, Theorem 3.1, gives the optimal strategy as the local time process of a diffusion reflected obliquely at a curved free boundary in $\mathbb{R}^{2}$, the state space being the impact level and the holdings in the risky asset. The stochasticity of our optimal strategy arises from its adaptivity to the transient component of the price dynamics and is of local time type. In contrast to the models with additive price impact where the martingale part of the fundamental price is irrelevant for a risk-neutral trader, here the volatility of $\bar{S}$ is relevant, cf. Remark 3.3 .

We solve the singular control problem by explicitly constructing the value function as a classical solution of the HJB variational inequality. Our verification arguments differ from a more common approach (outlined in Remark 6.4) since we were not able to verify the optimality more directly, due to the technical complications arising from the implicit nature of the eigenfunctions of the infinitesimal generator for the OU process (see Remark 6.8). In contrast, we first restrict the set of optimization strategies to those described by diffusions reflected at monotone boundaries, and optimize over the set of possible boundaries. To be able to apply methods from calculus of variations, 
we derive an explicit formula (eq. 4.6) for the Laplace transform of the inverse local times of diffusions reflected at elastic boundaries, i.e. boundaries which vary with the local time that the reflected process has spent at the boundary, and employ a change of coordinates. By solving the calculus of variations problem, we construct the candidate optimal free boundary and, moreover, show (one-sided) local optimality in the sense of Theorem 5.6. The latter is crucial for our verification of optimality.

The paper is organized as follows. Section 3 states the solution for the singular stochastic control problem posed in Section 2, and outlines the general course of arguments to come. In Section 4, a calculus of variations problem is posed, by restricting to strategies given by diffusions reflected at smooth boundaries. The free boundary is thereby constructed in Section 5. By solving the HJB variational inequality (3.3), we prove optimality and derive the value function and the optimal control in Section 6 .

\section{The model and the optimal control problem}

We consider a filtered probability space $\left(\Omega, \mathcal{F},\left(\mathcal{F}_{t}\right)_{t>0}, \mathbb{P}\right)$ with two correlated Brownian motions $W$ and $B$ with correlation coefficient $\rho \in[-1,1]$, such that

$$
[W, B]_{t}=\rho t, \quad t \geq 0 .
$$

for the quadratic co-variation of $W$ and $B$. The filtration $\left(\mathcal{F}_{t}\right)_{t \geq 0}$ is assumed to satisfy the usual conditions of completeness and right continuity, so we can take càdlàg versions for semimartingales. For notions from stochastic analysis we refer to JS03.

We consider a market with a risky asset, in addition to the riskless numéraire asset whose (discounted) price is constant at 1 . The large investor holds $\Theta_{t} \geq 0$ shares of the risky asset at time $t$. She may liquidate her initial position of $\Theta_{0-}$ shares by trading according to

$$
\Theta_{t}:=\Theta_{0-}-A_{t},
$$

where $A$ is a predictable, càdlàg, monotone process, describing the cumulative number of assets sold up to time $t$. We define the set of admissible strategies as

$$
\begin{gathered}
\mathcal{A}\left(\Theta_{0-}\right):=\{A: A \text { non-decreasing, càdlàg, predictable, } \\
\text { with } \left.0=: A_{0-} \leq A_{t} \leq \Theta_{0-}\right\} .
\end{gathered}
$$

The unaffected fundamental price $\bar{S}=\left(\bar{S}_{t}\right)_{t \geq 0}$ of the risky asset evolves according to

$$
\mathrm{d} \bar{S}_{t}=\mu \bar{S}_{t} \mathrm{~d} t+\sigma \bar{S}_{t} \mathrm{~d} W_{t}, \quad \bar{S}_{0} \in(0, \infty), \text { with } \sigma>0, \mu \in \mathbb{R},
$$

as a geometric Brownian motion, in the absence of perturbations by large investor trading. By trading, however, the large investor has market impact on the actual price

$$
S_{t}:=f\left(Y_{t}\right) \bar{S}_{t},
$$

of the risky asset through some impact process $Y$, by an increasing positive smooth function $f>0$ with $f(0)=1$. The process $Y$ can be interpreted as a volume effect 
process, representing the transient volume displacement by large trades in a limit order book (LOB) whose shape corresponds to the price impact function $f$, see Remark 2.3 . For $\hat{\sigma}>0$ the effect from perturbations $\hat{\sigma} \mathrm{d} B_{t}-\mathrm{d} A_{t}$ on the process

$$
\mathrm{d} Y_{t}=-\beta Y_{t} \mathrm{~d} t+\hat{\sigma} \mathrm{d} B_{t}-\mathrm{d} A_{t}, \quad Y_{0-}=y,
$$

is transient over time, in that $Y$ is mean reverting towards zero with mean reversion rate $\beta>0$. Existence and uniqueness of a strong solution to (2.3) are guaranteed for instance by PTW07, Thm. 4.1]. Sometimes we shall write $Y^{y, A}$ to stress the dependence of $Y$ on its initial state $y$ and the strategy $A$. The dynamics of $Y$ are of Ornstein-Uhlenbeck type, driven by $\hat{\sigma} \mathrm{d} B-\mathrm{d} A$. The mean-reversion property of the OU process has the financial interpretation that in the absence of activity from the large trader, the impact lessens since $Y$ reverts back to the neutral state zero and hence the price recovers to the fundamental price $\bar{S}$, thus modeling the transient component of the impact (in absolute terms).

For $\gamma \geq 0$, the $\gamma$-discounted proceeds up to time $t$ from a liquidation strategy $A$ are

$$
L_{t}(y ; A):=\int_{0}^{t} e^{-\gamma u} f\left(Y_{u}\right) \bar{S}_{u} \mathrm{~d} A_{u}^{c}+\sum_{\substack{0 \leq u \leq t \\ \Delta A_{u} \neq 0}} e^{-\gamma u} \bar{S}_{u} \int_{0}^{\Delta A_{u}} f\left(Y_{u-}-x\right) \mathrm{d} x,
$$

for $t \geq 0$, where $A_{t}=A_{t}^{c}+\sum_{u \leq t} \Delta A_{u}$ is the (pathwise) decomposition of $A$ into its continuous and pure-jump part, and $Y=Y^{y, A}$ solves (2.3). Jump terms in (2.4) can be justified from a LOB perspective (cf. Remark 2.3 below) or by stability results, see BBF17b, Sect. 5.3] for details. In particular, if $A^{n} \rightarrow A$ converges in the Skorokhod $M_{1}$ topology in probability for, e.g., continuous strategies $A^{n}$ and possibly non-continuous $A$, then the above definition ensures that $L\left(y ; A^{n}\right) \rightarrow L(y ; A)$ in $M_{1}$ in probability.

As $L$ is an increasing process, the limit $L_{\infty}:=\lim _{t \rightarrow \infty} L_{t}$ exists. The large trader's objective is to maximize expected (discounted) proceeds over an infinite time horizon,

$$
\max _{A \in \mathcal{A}\left(\Theta_{0-}\right)} \mathbb{E}\left[L_{\infty}(y ; A)\right] \quad \text { with } \quad v(y, \theta):=\sup _{A \in \mathcal{A}(\theta)} \mathbb{E}\left[L_{\infty}(y ; A)\right],
$$

where $v(y, \theta)$ denotes the value function for $y \in \mathbb{R}$ and $\theta \in[0, \infty)$.

Remark 2.1. The value function $v$ is increasing in $y$ and $\theta$. Indeed, monotonicity in $\theta$ follows from $\mathcal{A}\left(\theta_{1}\right) \subset \mathcal{A}\left(\theta_{2}\right)$ for $\theta_{1} \leq \theta_{2}$. For monotonicity in $y$, note that for $y_{1} \leq y_{2}$ and any strategy $A \in \mathcal{A}(\theta)$ one has $Y_{t}^{y_{1}, A} \leq Y_{t}^{y_{2}, A}$ for all $t$, implying $L_{t}\left(y_{1} ; A\right) \leq L_{t}\left(y_{2} ; A\right)$.

For the rest of the paper, the function $f$ and scalars $\beta, \mu, \gamma, \sigma, \rho, \hat{\sigma}$ satisfy

\section{Assumption 2.2.}

C1. We have $\delta:=\gamma-\mu>0$, that means the drift coefficient $-\delta \bar{S}$ for the $\gamma$-discounted fundamental price $e^{-\gamma t} \bar{S}_{t}$ is negative.

C2. The impact function $f \in C^{3}(\mathbb{R})$ satisfies $f, f^{\prime}>0$ and $\left(f^{\prime} / f\right)^{\prime}<\left(\Phi^{\prime} / \Phi\right)^{\prime}$, where

$$
\Phi(x):=\Phi_{\delta}(x):=H_{-\delta / \beta}((\sigma \rho \hat{\sigma}-\beta x) /(\sqrt{\beta} \hat{\sigma}))
$$

with Hermite function $H_{\nu}$ (cf. Leb72, Sect. 10.2]) and $\sigma, \hat{\sigma}, \beta>0$ and $\rho \in[-1,1]$. 
C3. The impact function $f$ furthermore satisfies $\left(f^{\prime} / f\right)^{\prime}<\left(\Phi^{\prime \prime} / \Phi^{\prime}\right)^{\prime}$.

C4. The function $\lambda(y):=f^{\prime}(y) / f(y), y \in \mathbb{R}$, is bounded, i.e. there exists $\lambda_{\max } \in(0, \infty)$ such that $0<\lambda(y) \leq \lambda_{\max }$ for all $y \in \mathbb{R}$.

C5. The function $k(y):=\frac{\hat{\sigma}^{2}}{2} \frac{f^{\prime \prime}(y)}{f(y)}-(\beta+\delta)+(\sigma \rho \hat{\sigma}-\beta y) \frac{f^{\prime}(y)}{f(y)}$ is strictly decreasing.

C6. There exist $y_{0}$ and $y_{\infty} \in \mathbb{R}$ such that $\left(f^{\prime} / f\right)\left(y_{0}\right)=\left(\Phi^{\prime} / \Phi\right)\left(y_{0}\right)$ and $\left(f^{\prime} / f\right)\left(y_{\infty}\right)=\left(\Phi^{\prime \prime} / \Phi^{\prime}\right)\left(y_{\infty}\right)$ holds.

Assumption 2.2 is satisfied by e.g. $f(y)=\exp (\lambda y)$ with $\lambda \in(0, \infty)$, cf. Lemma 5.1 below. See [BBF17a, Section 2.1] for the shape of the related multiplicative LOB. Note that $\Phi$ is (up to a constant factor) the unique positive and increasing solution of the ODE $\frac{\hat{\sigma}^{2}}{2} \Phi^{\prime \prime}(y)+(\sigma \rho \hat{\sigma}-\beta y) \Phi^{\prime}(y)-\delta \Phi(y)=0$.

The overall negative drift in Assumption C1 ensures that the optimization problem on an infinite time horizon has a finite value. Assumptions C2 and C3 imply uniqueness of the (boundary) points $y_{0}$ and $y_{\infty}$ from Assumption C6 which are needed in Lemma 5.3 While C3, uniqueness of $y_{\infty}$, is not crucial there, it will be needed in (6.14) for the verification. The bound on $\lambda$ in Assumption C4 is used to show some growth condition on the value function in Lemma 6.5 that is required to apply the martingale optimality principle (Proposition 6.1). Assumption C5 is needed for the verification Lemma 6.7.

Let us now comment on the model and its financial interpretation.

Remark 2.3. We explain how the price impact function $f$ can be interpreted in terms of a (static) multiplicative limit order book (LOB) and $Y$ can be viewed as a volume effect process in spirit of [PSS11, which in our context relates the relative price impact to transient imbalances of volume. To this end, let us recall the connection between price impact function $f$ and the (general) density of a LOB, see BBF17a, Sect. 2.1] for more detail and examples. For relative price perturbations $r_{t}:=S_{t} / \bar{S}_{t}$, let $q(r) \mathrm{d} r$ denote the density of offers available at price $r \bar{S}_{t}$. We call the (signed) measure induced by $q$ the multiplicative limit order book. Its cumulative distribution function is $Q(r):=\int_{1}^{r} q(x) \mathrm{d} x$. The total volume of assets available for prices in some (interval) range $\left\{r \bar{S}_{t}: r \in R\right\}$ with measurable $R \subset(0, \infty)$ is $\int_{R} q(x) \mathrm{d} x$. So, a block sale of size $\Delta A_{t}>0$ at time $t$ moves the price from $r_{t-} \bar{S}_{t}$ to $r_{t} S_{t}$ such that the volume changes according to $Q\left(r_{t}\right)=Q\left(r_{t-}\right)-\Delta A_{t}$, giving (discounted) proceeds $e^{-\gamma t} \bar{S}_{t} \int_{r_{t}}^{r_{t-}} x \mathrm{~d} Q(x)$. In the terminology of Kyl85, $Q\left(r_{t}\right)-Q\left(r_{t-}\right)$ reflects the depth of the LOB for price changes by a factor of $r_{t} / r_{t-}$. A change of variables with $Y_{t}:=Q\left(r_{t}\right)$ and $f:=Q^{-1}$ yields the jump term in 2.4. In this sense, $Y$ denotes the effect from the past and present trades on the volume displacement in the LOB. By the drift in (2.3), this effect is persistent over time but not permanent. Its transient nature relates to the liquidity property that Kyl85] calls resilience. Note that in our model the resilience is stochastic in the sense that the volume effect process $Y$ in 2.3 is, whereas the resilience rate $\beta$ is constant (differently e.g. to GH16]).

Remark 2.4. Stochasticity may account for variations of transient impact that cannot be entirely explained by the single agent's own trading activity, and thus not solely described by deterministic functional modeling. 
(a) Most of the literature on transient impact considers impact that is a deterministic function of the actions of a single large trader. We consider here an application problem for an individual large trader, but we do not want to assume that she is the only large trader in the market, or that she is as an aggregate of all large traders (a possibility mentioned in [Fre98]). The additional stochastic noise term $\hat{\sigma} \mathrm{d} B_{t}$ in 2.3 can be understood as the aggregate influence on the impact by other large 'noise' traders (acting non-strategically). Questions on strategic behavior between multiple traders (like in [SZ17]) are interesting but beyond the present paper.

(b) Note that the volatility and as well the drift of the (marginal) price process $S=f\left(Y_{t}\right) \bar{S}_{t}$ from 2.2 , at which (additional infinitesimally) small quantities of the risky assets would be traded, are stochastic via the additional stochastic component of $Y$. Furthermore, we emphasize that the form of relative price impact function $\Delta \mapsto f\left(Y_{t-}+\Delta\right) / f\left(Y_{t-}\right)$ can vary with $Y$ in general. In the sense of Remark 2.3, this means the general shape of the corresponding LOB can exhibit stochastic variations from the large trader's perspective.

(c) Recently, LN17 suggested to model a signal, which predicts the short-term evolution of prices, as an Ornstein-Uhlenbeck process that modulates the drift of the price dynamics. One can interpret stochasticity of $Y$ as such a signal as follows. For $\lambda=f^{\prime} / f$ being constant, the $\log$-price can be written as $\log S=\left(\log \bar{S}+\lambda Y^{\mathrm{sig}}\right)+\lambda Y^{\operatorname{trans}, \Theta}$, where $Y^{\mathrm{sig}}$ is a mean-reverting signal with $\mathrm{d} Y_{t}^{\mathrm{sig}}=-\beta Y_{t}^{\mathrm{sig}} \mathrm{d} t+\hat{\sigma} \mathrm{d} B_{t}$ and $Y^{\operatorname{trans}, \Theta}$ is the transient impact from trading with $\mathrm{d} Y_{t}^{\text {trans, },}=-\beta Y_{t}^{\text {trans, }, \Theta} \mathrm{d} t+\mathrm{d} \Theta_{t}$. From this perspective, the optimal liquidation strategy will be adaptive to the signal and depend on the correlation between the signal and $\log \bar{S}$, see Theorem 3.1 and Remark 3.3 .

Remark 2.5. Noting that a bid-ask spread is not modeled explicitly and price impact $f$ (i.e. the LOB shape) is static, we consider the model as being at a mesoscopic level for low-frequency problems, rather than for market microstructure effects in high frequency. At this level and as pointed out in [AKS16, Rmk. 2.2], it is sensible to think of price impact and liquidity costs as being aggregated over various types of orders. The LOB from Remark 2.3 should be interpreted accordingly. Note that in this paper we deal with monotone strategies and thus only one (bid) side of the LOB is relevant. Considering infinite time horizon can be viewed as approximation for a longer horizon with more analytic tractability. Concerning the question of comparison with additive models for transient impact, positivity of asset prices is desirable from a theoretical point of view, relevant for applications with longer time horizons (as they may occur e.g. for large institutional trades, cf. e.g. [CL95, or for hedging problems with longer maturities), and appears to fit better to common models with multiplicative price evolutions like (2.1). See BBF17a, Example 5.4] for a more detailed discussion and further references.

\section{The optimal strategy and how it will be derived}

This section states the main theorem which describes the solution to the singular stochastic control problem, and outlines afterwards the general course of arguments 
for proving it in the subsequent sections. To explain ideas, let us first motivate how the variational inequality (3.3), being the dynamical programming equation for the optimization problem at hand, is readily suggested by an application of the martingale optimality principle. To this end, consider for an admissible strategy $A$ the process

$$
G_{t}(y ; A):=L_{t}(y ; A)+e^{-\gamma t} \bar{S}_{t} V\left(Y_{t}, \Theta_{t}\right),
$$

where $G_{0-}(y ; A)=\bar{S}_{0} V\left(Y_{0-}, \Theta_{0-}\right)$ and $V \in C^{2,1}(\mathbb{R} \times[0, \infty) ;[0, \infty))$ is some function. Suppose $V$ can be chosen such that $G$ is a supermartingale. Then one should have

$$
\begin{aligned}
\bar{S}_{0} V\left(y, \Theta_{0-}\right) & =\mathbb{E}\left[G_{0-}(y ; A)\right] \\
& \geq \lim _{T \rightarrow \infty} \mathbb{E}\left[L_{T}(y ; A)\right]+\lim _{T \rightarrow \infty} e^{-\gamma T} \mathbb{E}\left[\bar{S}_{T} V\left(Y_{T}, \Theta_{T}\right)\right] \\
& =\mathbb{E}\left[L_{\infty}(y ; A)\right]
\end{aligned}
$$

heuristically, provided that the second summand on the right-hand side converges to 0 . Hence, for $V$ being such that $G$ is a supermartingale for every admissible strategy $A$ and a martingale for at least one strategy $A^{*}$, one can conclude that $V$ is essentially the value function for (2.5) (modulo the factor $\bar{S}_{0}$ ). To describe $V$, one may apply Itô's formula to get

$$
\begin{aligned}
\mathrm{d} G_{t}=e^{-\gamma t} \bar{S}_{t} & \left(\hat{\sigma} V_{y}\left(Y_{t-}, \Theta_{t-}\right) \mathrm{d} B_{t}+\sigma V\left(Y_{t-}, \Theta_{t-}\right) \mathrm{d} W_{t}\right. \\
& +\left((\mu-\gamma) V+\left(\sigma \rho \hat{\sigma}-\beta Y_{t-}\right) V_{y}+\frac{\hat{\sigma}^{2}}{2} V_{y y}\right)\left(Y_{t-}, \Theta_{t-}\right) \mathrm{d} t \\
& +\left(f-V_{y}-V_{\theta}\right)\left(Y_{t-}, \Theta_{t-}\right) \mathrm{d} A_{t}^{c} \\
& \left.+\int_{0}^{\Delta A_{t}}\left(f-V_{y}-V_{\theta}\right)\left(Y_{t-}-x, \Theta_{t-}-x\right) \mathrm{d} x\right) .
\end{aligned}
$$

Define, with $\delta=\gamma-\mu$, a differential operator on $C^{2,0}$ functions $\varphi$ by

$$
\mathcal{L} \varphi(y, \theta):=\frac{\hat{\sigma}^{2}}{2} \varphi_{y y}(y, \theta)+(\sigma \rho \hat{\sigma}-\beta y) \varphi_{y}(y, \theta)-\delta \varphi(y, \theta) .
$$

By equation (3.2), solving the Hamilton-Jacobi-Bellman (HJB) variational inequality

$$
0=\max \left\{f-V_{y}-V_{\theta}, \mathcal{L} V\right\} \quad \text { with } V(y, 0)=0, y \in \mathbb{R},
$$

would suffice for $G$ to be a local (super-)martingale. This suggests the existence of a sell region $\mathcal{S}$ (action region) where the $\mathrm{d} A$-integrand $f-V_{y}-V_{\theta}$ is zero and it is optimal to trade (i.e. sell), and a wait region $\mathcal{W}$ (inaction region) in which the $\mathrm{d} t$-integrand $\mathcal{L} V$ is zero and it is optimal not to trade. Assume that the two regions

$$
\begin{aligned}
\mathcal{S} & =\{(y, \theta) \in \mathbb{R} \times(0, \infty): \mathbb{y}(\theta)<y\} \quad \text { and } \\
\mathcal{W} & =\{(y, \theta) \in \mathbb{R} \times(0, \infty): y<\mathbb{y}(\theta)\}
\end{aligned}
$$

are separated by a free boundary $\{(y, \theta): y=\mathbb{y}(\theta)\}$. An optimal strategy, i.e. a strategy for which $G$ is a martingale, would be described as follows: if $\left(Y_{0-}, \Theta_{0-}\right) \in \mathcal{S}$, then 
perform a block sale of size $\Delta A_{0}$ such that $\left(Y_{0}, \Theta_{0}\right)=\left(Y_{0-}-\Delta A_{0}, \Theta_{0-}-\Delta A_{0}\right) \in \partial \mathcal{S}$. Thereafter, if $\Theta_{0}>0$, sell just enough as to keep the process $(Y, \Theta)$ within $\overline{\mathcal{W}}$. In this way, the process $(Y, \Theta)$ should be described by a diffusion process that is reflected at the boundary $\partial \mathcal{W} \cap \partial \mathcal{S}$ in direction $(-1,-1)$, i.e. there is waiting in the interior and selling at the boundary until all shares are sold, when $(Y, \Theta)$ hits $\{(y, 0): \mathbb{y}(0) \leq y\}$. For such reflected diffusions, exsistence and uniqueness follow from classical results, see Remark 4.2 , and Theorem 4.3 provides important characteristics which are key to the subsequent construction of the optimal control. The solution of the optimal liquidation problem is indeed described by the local time process of a diffusion reflected at a boundary which is explicitly given by an ODE. This main result is stated as Theorem 3.1 below.

In the following sections, we will find the value function for our stochastic control problem by constructing a classical solution of the variational inequality (3.3). Provided that the key variational inequalities for the (candidate) solution are satisfied, optimality can be verified by typical martingale arguments, see Proposition 6.1. Based on results on reflected diffusions from Theorem 4.3, we reformulate in Section 4 the optimization problem as a (nonstandard) calculus of variations problem. Its solution, derived in Section 5, provides a candidate for the free boundary, separating the regions of action and inaction, together with the value function on that boundary. Moreover, we show a (one-sided) local optimality property of the derived boundary (cf. Theorem 5.6). This will be crucial in Section 6 (cf. proof of Lemma 6.7) to verify (3.3) for the candidate value function, constructed there, in order to finally conclude on p. 27 the proof for

Theorem 3.1. Let Assumption 2.2 be satisfied. Then the ordinary differential equation

$$
\mathbb{y}^{\prime}(\theta)=\left(\frac{\left(\left(\Phi^{\prime}\right)^{2}-\Phi \Phi^{\prime \prime}\right)\left(f^{\prime} \Phi^{\prime}-f \Phi^{\prime \prime}\right) / \Phi}{\left(\Phi \Phi^{\prime \prime}-\left(\Phi^{\prime}\right)^{2}\right) f^{\prime \prime}+\left(\Phi^{\prime} \Phi^{\prime \prime}-\Phi \Phi^{\prime \prime \prime}\right) f^{\prime}+\left(\Phi^{\prime} \Phi^{\prime \prime \prime}-\left(\Phi^{\prime \prime}\right)^{2}\right) f}\right)(\mathbb{y}(\theta))
$$

with initial condition $\mathrm{y}(0)=y_{0}$ admits a unique solution $\mathrm{y}:[0, \infty) \rightarrow \mathbb{R}$, that is strictly decreasing and maps $[0, \infty)$ bijectively to $\left(y_{\infty}, y_{0}\right]$, for $y_{0}$ and $y_{\infty}$ from Assumption C6.

The boundary function $\mathbb{y}$ characterizes the solution of problem 2.5 as $A^{*}=(\Delta+K) \mathbb{1}_{\llbracket 0, \tau \rrbracket}$,

where $\Delta:=\Theta_{0-} \mathbb{1}_{\left\{Y_{0-} \geq y_{0}+\Theta_{0-}\right\}}+\tilde{\Delta} \mathbb{1}_{\left\{Y_{0-}<y_{0}+\Theta_{0-}, \tilde{\Delta} \geq 0\right\}}$ with $\tilde{\Delta} \leq \Theta_{0-}$ satisfying $Y_{0-}-\tilde{\Delta}=\mathrm{y}\left(\Theta_{0-}-\tilde{\Delta}\right)$, and where $(Y, K)$ is the unique continuous adapted process on $\llbracket 0, \tau \rrbracket$ with non-decreasing $K$ which solves the $\mathbb{y}$-reflected $S D E$

$$
\begin{aligned}
Y_{t} & \leq \mathbb{y}\left(\Theta_{0-}-\Delta-K_{t}\right), \\
\mathrm{d} Y_{t} & =-\beta Y_{t} \mathrm{~d} t+\hat{\sigma} \mathrm{d} B_{t}-\mathrm{d} K_{t}, \\
\mathrm{~d} K_{t} & =\mathbb{1}_{\left\{Y_{t}=\mathrm{y}\left(\Theta_{0-}-\Delta-K_{t}\right)\right\}} \mathrm{d} K_{t},
\end{aligned}
$$

starting in $\left(Y_{0-}-\Delta, 0\right)$, for time to liquidation $\tau:=\inf \left\{t \geq 0: K_{t}=\Theta_{0-}-\Delta\right\}$.

Moreover, $\tau$ has finite moments.

Remark 3.2. The optimal control $A^{*}$ acts as follows: 1) If $Y_{0-} \geq y_{0}+\Theta_{0-}$, sell everything immediately at time 0 and stop trading; 2) Otherwise, if $\left(\Theta_{0-}, Y_{0-}\right)$ is such that $\mathrm{y}\left(\Theta_{0_{-}}\right)<Y_{0-}<y_{0}+\Theta_{0_{-}}$, perform an initial block trade of size $A_{0}^{*}:=\Delta>0$ so that $Y_{0}=Y_{0-}-\Delta$ is on the boundary $Y_{0}=\mathbb{y}\left(\Theta_{0}\right)$. Now being in the wait region 
$\overline{\mathcal{W}}$, sell as much as to keep with the least effort the state process $(Y, \Theta)$ in $\overline{\mathcal{W}}$ until all assets are liquidated at time $\tau$ (cf. Figure 1 , waiting e.g. at times $t \in[25,34]$ since then impact $Y_{t}$ is less than $\left.\mathrm{y}\left(\Theta_{t}\right)\right)$.

The inverse local time $\tau_{\ell}:=\inf \left\{t>0: K_{t}>\ell\right\}$ is simply how long it takes to liquidate $\ell$ assets (after an initial block sale). For $\tau>0$ (case 2 in Remark 3.2 its Laplace transform is

$$
\mathbb{E}\left[e^{-\alpha \tau_{\ell}}\right]=\frac{\Phi_{\alpha}\left(Y_{0}\right)}{\Phi_{\alpha}\left(\mathrm{y}\left(\Theta_{0}\right)\right)} \exp \left(\int_{0}^{\ell}\left(\mathrm{y}^{\prime}\left(\Theta_{0}-x\right)+1\right) \frac{\Phi_{\alpha}^{\prime}\left(\mathrm{y}\left(\Theta_{0}-x\right)\right)}{\Phi_{\alpha}\left(\mathrm{y}\left(\Theta_{0}-x\right)\right)} \mathrm{d} x\right)
$$

for $\alpha>0$ and $0 \leq \ell \leq \Theta_{0}=\Theta_{0-}-\Delta$, as it will be shown in the proof of Theorem 3.1. Using analyticity of $\Phi_{\alpha}$ w.r.t. the parameter $\alpha$, one easily gets that $\tau_{\ell}$ has finite moments. Moreover, the Laplace transform (3.5) gives access to the distribution of the time to liquidation $\tau$ by efficient numerical inversion, as in e.g. AW95.

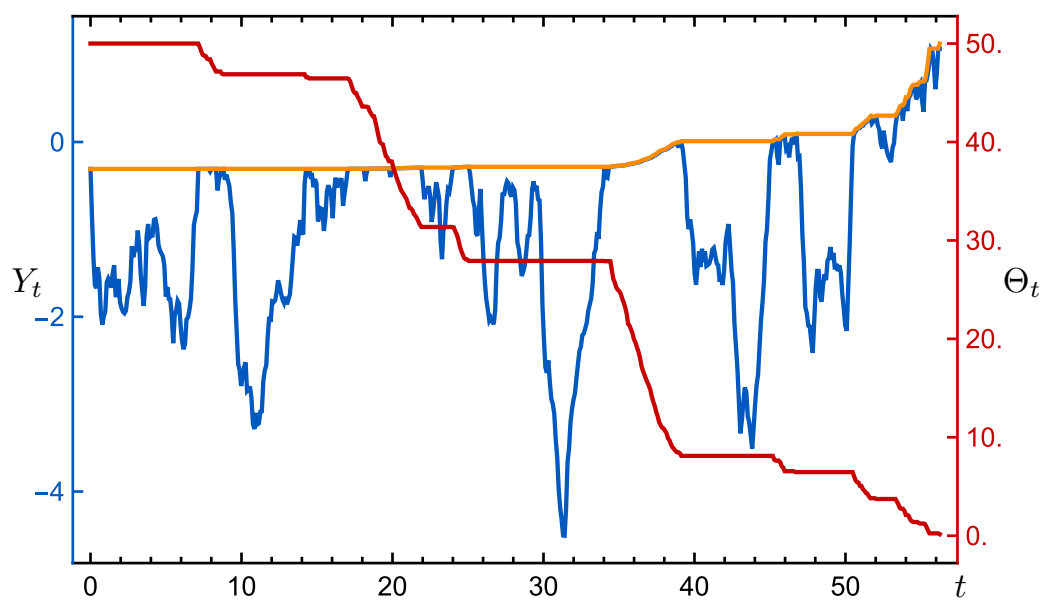

Figure 1: Sample path of impact $Y_{t}$ (blue), asset position $\Theta_{t}$ (red, decreasing) and reflecting boundary $\mathrm{y}\left(\Theta_{t}\right)$ (orange, increasing) for optimally liquidating $\Theta_{0}=50$ assets (after an initial block trade $\Delta$ ), with $\delta=0.1, \beta=1, \rho=0$, $\hat{\sigma}=1$ and $f(\cdot)=\exp (\cdot)$.

Remark 3.3. The optimizer depends on the volatility of the fundamental price. If correlation $\rho$ is not zero, the optimal strategy and the shape of the free boundary do depend on the volatility $\sigma$ of the fundamental price process. This is a notable difference to many additive impact models, where the optimal liquidation strategy does not depend on the martingale part of the fundamental price process, cf. e.g. [LS13, Sect. 2.2]. To stress the dependence on $\rho$, we write $\Phi^{\rho}$ for $\Phi$ in (2.6), denote by $F^{\rho}$ the right-hand side of (3.4) and by $y_{0}^{\rho}$ the root of $f^{\prime} / f-\left(\Phi^{\rho}\right)^{\prime} / \Phi^{\rho}$. So the solution $\mathrm{y}^{\rho}$ of the $\operatorname{ODE}\left(\mathrm{y}^{\rho}\right)^{\prime}(\theta)=F^{\rho}\left(\mathrm{y}^{\rho}(\theta)\right)$ with $\mathrm{y}^{\rho}(0)=y_{0}^{\rho}$ is the optimal boundary function from Theorem 3.1. In the special case of constant $\lambda$, i.e. $f(y)=e^{\lambda y}$, we have 
$F^{\rho}(y)=F^{0}(y-\sigma \rho \hat{\sigma} / \beta)$ since $\Phi^{\rho}(y)=\Phi^{0}(y-\sigma \rho \hat{\sigma} / \beta)$, and thus $\mathbb{y}^{\rho}(\theta)=\mathbb{y}^{0}(\theta)+\sigma \rho \hat{\sigma} / \beta$. For general $f$, investigating $y_{0}$ and $y_{\infty}$ from Assumption C6 still reveals a similar displacement of the boundary. Thus, when impact and fundamental price are positively correlated $(\rho>0)$, it is optimal to trade slower if fundamental price volatility is larger, since the wait region increases.

Remark 3.4. The optimal liquidation problem with deterministic impact dynamics $(\hat{\sigma}=0)$ is solved in BBF17a, Thm. 3.4] and characterized by an optimal boundary function $\mathrm{y}^{0}$. Assumption 2.2 implies the model assumptions BBF17a, Assumption 3.2] of that theorem. Using the asymptotic expansion [Leb72, eq. (10.6.6)] of Hermite functions, straightforward calculations show uniform convergence $\left\|\mathrm{y}^{\hat{\sigma}}-\mathrm{y}^{0}\right\|_{\infty} \rightarrow 0$ of the boundaries as $\hat{\sigma} \searrow 0$, for $\mathrm{y}^{\hat{\sigma}}$ solving the ODE (3.4).

\section{Reformulation as a calculus of variations problem}

In this section we will recast the free boundary problem of the variational inequality (3.3) as a (nonstandard, at first) calculus of variations problem. To sketch the idea, suppose that the large trader has to liquidate $\Theta_{0} \geq 0$ shares and that $\left(Y_{0}, \Theta_{0}\right)$ is already on the free boundary between sell and wait regions (after an initial jump or waiting). Let $\mathbb{y}:\left[0, \Theta_{0}\right] \rightarrow \mathbb{R}$ be a $C^{1}$ function with $\mathbb{y}\left(\Theta_{0}\right)=Y_{0}$ and $\mathbb{y}^{\prime}<0$ (we expect the optimal boundary to be such). To find the optimal boundary curve $\mathbb{y}$, we will optimize expected proceeds over the set of $y$-reflected strategies $A:=A^{\text {refl }}\left(\mathbb{y}, \Theta_{0}\right)$ from

Definition 4.1. Let $(Y, A)$ be the (unique) pair of continuous adapted processes with non-decreasing $A$ such that $Y_{t} \leq \mathbb{y}\left(\Theta_{0}-A_{t}\right)$ and

$$
\begin{aligned}
\mathrm{d} Y_{t} & =-\beta Y_{t} \mathrm{~d} t+\hat{\sigma} \mathrm{d} B_{t}-\mathrm{d} A_{t}, & & Y_{0}=\mathrm{y}\left(\Theta_{0}\right), \\
\mathrm{d} A_{t} & =\mathbb{1}_{\left\{Y_{t}=\mathrm{y}\left(\Theta_{0}-A_{t}\right)\right\}} \mathrm{d} A_{t}, & & A_{0}=0,
\end{aligned}
$$

on $\llbracket 0, \tau \rrbracket$ for $\tau:=\inf \left\{t \geq 0: A_{t}=\Theta_{0}\right\}$. We call $A^{\text {refl }}\left(\mathbb{y}, \Theta_{0}\right):=A$ a $\mathbb{y}$-reflected strategy.

Remark 4.2. Existence and uniqueness of a strong solution $(Y, A)$ follows from (a careful extension of) classical results, cf. [DI93], by considering the pair $(Y, A)$ as a (degenerate) diffusion in $\mathbb{R}^{2}$ with oblique direction of reflection $(-1,+1)$ at a smooth boundary. Considered as a one-dimensional diffusion, the process $Y$ is reflected at a boundary that moves with its local time $A$. In this sense, we call the reflection elastic.

Viewing $Y$ as a diffusion with reflection at $\mathrm{y}$, we can rewrite expected proceeds from $A$ as a deterministic functional of $\mathbb{y}$, see (4.8) below, whose maximizer should describe the optimal strategy. For this step we rely crucially on a representation for the Laplace transform of the inverse local time of reflected diffusions from Theorem 4.3 . Since the integrand of 4.8 depends on the whole path $\mathbb{y}$, a reparametrization is necessary to obtain a tractable calculus of variations problem 4.10 - 4.11).

Let $\tau_{\Theta_{0}}$ be the stopping time when $A=\Theta_{0}$. For the continuous y-reflected strategy 
$A$ with proceeds $L:=L\left(\mathbb{y}\left(\Theta_{0}\right) ; A\right)$, we have by $\mathrm{DM} 82$, Theorem 57$]$ for any $T \in[0, \infty)$,

$$
\begin{aligned}
\mathbb{E}\left[L_{T}\right] & =\mathbb{E}\left[\int_{0}^{\tau_{\Theta_{0}} \wedge T} f\left(Y_{t}\right) e^{-\delta t} \mathcal{E}(\sigma W)_{t} \mathrm{~d} A_{t}\right] \\
& =\mathbb{E}\left[\mathcal{E}(\sigma W)_{T} \int_{0}^{\tau_{\Theta_{0}} \wedge T} f\left(Y_{t}\right) e^{-\delta t} \mathrm{~d} A_{t}\right] .
\end{aligned}
$$

For fixed $T$, let $\mathbb{Q}$ be the measure given by $\mathrm{d} \mathbb{Q} / \mathrm{d} \mathbb{P}=\mathcal{E}(\sigma W)_{T}$ on $\mathcal{F}_{T}$. Then

$$
\mathbb{E}\left[L_{T}\right]=\mathbb{E}^{\mathbb{Q}}\left[\int_{0}^{\tau_{\Theta_{0}} \wedge T} f\left(Y_{t}\right) e^{-\delta t} \mathrm{~d} A_{t}\right]
$$

Girsanov's theorem gives that the process $\widetilde{B}_{t}:=B_{t}-[B, \sigma W]_{t}=B_{t}-\sigma \rho t$ is a Brownian motion under $\mathbb{Q}$. Therefore, we have under $\mathbb{Q}$

$$
\mathrm{d} Y_{t}=\left(\sigma \rho \hat{\sigma}-\beta Y_{t}\right) \mathrm{d} t+\hat{\sigma} \mathrm{d} \widetilde{B}_{t}-\mathrm{d} A_{t},
$$

i.e. the impact process $Y$ is a (reflected) Ornstein-Uhlenbeck process with shifted (non-zero) mean reversion level, and $A$ is its local time on the boundary. We cannot directly pass to the limit $T \rightarrow \infty$ in (4.1) because the measure change $\mathbb{Q}$ depends on $T$. However, note that the right-hand side of (4.1) depends only on the law of the reflected diffusion $(Y, A)$ under the measure $\mathbb{Q}$. That is why we consider the reflected diffusion $\left(X, A^{X}\right)$ with the following dynamics under $\mathbb{P}$ : for $g(a):=\mathrm{y}\left(\Theta_{0}-a\right)$ let

$$
\begin{aligned}
\mathrm{d} X_{t} & =\left(\sigma \rho \hat{\sigma}-\beta X_{t}\right) \mathrm{d} t+\hat{\sigma} \mathrm{d} B_{t}-\mathrm{d} A_{t}^{X}, & & X_{0}=g(0), \\
\mathrm{d} A_{t}^{X} & =\mathbb{1}_{\left\{X_{t}=g\left(A_{t}^{X}\right)\right\}} \mathrm{d} A_{t}^{X}, & & A_{0}^{X}=0, \\
\tau_{\ell}^{X} & :=\inf \left\{t>0: A_{t}^{X}>\ell \text { or } A_{t}^{X}=\Theta_{0}\right\}, & &
\end{aligned}
$$

such that in addition $X_{t} \leq g\left(A_{t}^{X}\right)$, on $\llbracket 0, \tau_{\Theta_{0}}^{X} \rrbracket$. Existence and uniqueness of a strong solution $\left(X, A^{X}\right)$ until $\tau_{\Theta_{0}}^{X}$ follows as in Remark 4.2 .

Now, by 4.1) we have $\mathbb{E}\left[L_{T}\right]=\mathbb{E}\left[\int_{0}^{\tau_{\Theta_{0}}^{X} \wedge T} f\left(X_{t}\right) e^{-\delta t} \mathrm{~d} A_{t}^{X}\right]$, which gives for $T \rightarrow \infty$ by monotone convergence on both sides

$$
\begin{aligned}
\mathbb{E}\left[L_{\infty}\right] & =\mathbb{E}\left[\int_{0}^{\tau_{\Theta_{0}}^{X}} f\left(X_{t}\right) e^{-\delta t} \mathrm{~d} A_{t}^{X}\right]=\mathbb{E}\left[\int_{0}^{\tau_{\Theta_{0}}^{X}} f\left(g\left(A_{t}^{X}\right)\right) e^{-\delta t} \mathrm{~d} A_{t}^{X}\right] \\
& =\mathbb{E}\left[\int_{0}^{\Theta_{0}} f(g(\ell)) e^{-\delta \tau_{\ell}^{X}} \mathrm{~d} \ell\right]=\int_{0}^{\Theta_{0}} f(g(\ell)) \mathbb{E}\left[e^{-\delta \tau_{\ell}^{X}}\right] \mathrm{d} \ell,
\end{aligned}
$$

using (4.3). To express the latter as a functional of the free boundary only, we need

Theorem 4.3. The Laplace transform of $\tau_{\ell}^{X}$ from 4.2 -4.4 for $\Theta_{0}=\theta$ is

$$
\mathbb{E}\left[e^{-\delta \tau_{\ell}^{X}}\right]=\exp \left(\int_{\theta-\ell}^{\theta}\left(\mathbb{y}^{\prime}(x)-1\right) \frac{\Phi_{\delta}^{\prime}(\mathbb{y}(x))}{\Phi_{\delta}(\mathbb{y}(x))} \mathrm{d} x\right) \quad \text { for } \ell<\theta .
$$


Proof. We will identify the Laplace transform by calculating the terms in (4.5) at first for $f$ being replaced with arbitrary test functions $\varphi$, and then using ideas from calculus of variations. To identify $q(y, \theta):=\mathbb{E}\left[\int_{0}^{T} e^{-\delta t} \varphi\left(X_{t}\right) \mathrm{d} A_{t}^{X}\right]$ for continuous functions $\varphi: \mathbb{R} \rightarrow[0, \infty)$ with $X_{0}=y \leq \mathbb{y}(\theta), \Theta_{0}=\theta$ and $T:=\tau_{\theta}^{X}$, it suffices to construct $q$ such that

$$
M_{t}:=\int_{0}^{t} e^{-\delta u} \varphi\left(X_{u}\right) \mathrm{d} A_{u}^{X}+e^{-\delta t} q\left(X_{t}, \theta-A_{t}^{X}\right)
$$

is a martingale on $\llbracket 0, T \rrbracket$ with $e^{-\delta t} q\left(X_{t}, \theta-A_{t}^{X}\right) \rightarrow 0$ in $L^{1}$ as $t \rightarrow T$. Consider the state space $\mathcal{I}:=\{(y, \theta): y<\mathbb{y}(\theta)\}$. To check the martingale property, assuming that we have $q \in C^{2,1}(\mathcal{I}) \cap C^{1,1}(\overline{\mathcal{I}})$, Itô's formula yields (similarly to $(3.2)$ ) that $q_{y}+q_{\theta}=\varphi$ on $\partial \mathcal{I}$ and $\mathcal{L} q(y, \theta)=0$ in $\mathcal{I}$. Moreover, for $q$ increasing in $y$ we have $q(y, \theta)=\Phi(y) C(\theta)$ with $\Phi=\Phi_{\delta}$ from 2.6 and some function $C \in C^{1}$. Let $H(\theta):=q(\mathbb{y}(\theta), \theta)$. The condition $q_{y}+q_{\theta}=\varphi$ leads to

$$
H^{\prime}(\theta)=\Phi^{\prime}(\mathbb{y}(\theta)) C(\theta) \mathbb{y}^{\prime}(\theta)+\left(\varphi(\mathbb{y}(\theta))-\Phi^{\prime}(\mathbb{y}(\theta)) C(\theta)\right)=A(\theta) H(\theta)+B(\theta)
$$

where $A(\theta):=\left(\mathbb{y}^{\prime}(\theta)-1\right) \Phi^{\prime}(\mathbb{y}(\theta)) / \Phi(\mathbb{y}(\theta))$ and $B(\theta):=\varphi(\mathbb{y}(\theta))$. Solving this ODE for $H$ gives (since $H(0)=0$ )

$$
H(\theta)=\int_{0}^{\theta} \varphi(\mathbb{y}(z)) \exp \left(\int_{z}^{\theta}\left(\mathbb{y}^{\prime}(x)-1\right) \frac{\Phi_{\delta}^{\prime}(\mathbb{y}(x))}{\Phi_{\delta}(\mathbb{y}(x))} \mathrm{d} x\right) \mathrm{d} z,
$$

which yields the candidate $q(y, \theta)=\Phi(y) H(\theta) / \Phi(\mathbb{y}(\theta))$. It is straightforward to check $q \in C^{2,1}(\mathcal{I}) \cap C^{1,1}(\overline{\mathcal{I}})$ and $q_{y}+q_{\theta}=\varphi$ on $\partial \mathcal{I}$, giving that $M$ is a martingale, using boundedness of $q_{y}\left(X, \theta-A^{X}\right)$ on $\llbracket 0, T \rrbracket$. By monotonicity of $q$ in $y$, hence $q(y, \theta) \leq H(\theta)$, we obtain $e^{-\delta t} q\left(X_{t}, \theta-A_{t}^{X}\right) \rightarrow 0$ in $L^{1}$ as $t \rightarrow T$ via dominated convergence, so as in (4.5) we find

$$
\int_{0}^{\theta} \varphi(\mathbb{y}(z))(\underbrace{\mathbb{E}\left[e^{\left.-\delta \tau_{\theta-z}^{X}\right]}-\exp \left(\int_{z}^{\theta}\left(\mathbb{y}^{\prime}(x)-1\right) \frac{\Phi_{\delta}^{\prime}(\mathbb{y}(x))}{\Phi_{\delta}(\mathbb{y}(x))} \mathrm{d} x\right)\right.}_{=: \Delta(z)}) \mathrm{d} z=0 .
$$

Note that $z \mapsto \mathbb{E}\left[\exp \left(-\delta \tau_{\theta-z}^{X}\right)\right]$ is left-continuous. Hence, if $\Delta\left(z_{1}\right)>0$ for some $z_{1} \in(0, \theta]$, there exists $z_{0}<z_{1}$ such that $\Delta>0$ on $\left(z_{0}, z_{1}\right)$. Since $\mathrm{y}$ is bijective (recall that $\left.\mathrm{y}^{\prime}<0\right)$, we can find a continuous function $\varphi$ with $\varphi \circ \mathrm{y}>0$ inside $\left(z_{0}, z_{1}\right)$ and $\varphi \circ \mathrm{y}=0$ outside $\left(z_{0}, z_{1}\right)$, which yields $\int_{0}^{\theta} \varphi(\mathbb{y}(z)) \Delta(z) \mathrm{d} z>0$, contradicting 4.7. Similarly, $\Delta\left(z_{1}\right)<0$ also leads to a contradiction. Therefore $\Delta=0$ on $(0, \theta]$.

Remark 4.4. Let us note that Theorem 4.3 generalizes to general (regular) diffusions reflected at increasing boundaries by taking $\Phi_{\delta}$ to be the increasing non-negative $\delta$-eigenfunction of the generator of the diffusion. Indeed, the proof would not change.

Using Theorem 4.3 and 4.5 we derive the following representation for the proceeds from a y-reflected strategy in terms of the boundary:

$$
\mathbb{E}\left[L_{\infty}\right]=\int_{0}^{\Theta_{0}} f(g(\ell)) \exp \left(-\int_{0}^{\ell}\left(g^{\prime}(a)+1\right) \frac{\Phi_{\delta}^{\prime}(g(a))}{\Phi_{\delta}(g(a))} \mathrm{d} a\right) \mathrm{d} \ell .
$$


Since the $\mathrm{d} \ell$-integrand in 4.8 depends on the whole path of $g$, classical calculus of variations methods are not directly available. Since by definition $g(a)=\mathbb{y}\left(\Theta_{0}-a\right)$ we get with $\mathbb{r}(\ell):=\int_{0}^{\ell}\left(1-\mathbb{y}^{\prime}(x)\right) \frac{\Phi^{\prime}(\mathbb{y}(x))}{\Phi(\mathbb{y}(x))} \mathrm{d} x$ that

$$
\mathbb{E}\left[L_{\infty}\right]=e^{-\mathrm{r}\left(\Theta_{0}\right)} \int_{0}^{\Theta_{0}} f(\mathbb{y}(\ell)) e^{\mathrm{r}(\ell)} \mathrm{d} \ell .
$$

Since $\Phi^{\prime}, \Phi>0$ and $\mathrm{y}^{\prime}<0$, the function $\mathbb{r}$ in strictly increasing and thus has an inverse $\mathbb{r}^{-1}$. Fixing $R:=\mathbb{r}\left(\Theta_{0}\right)$ and setting $w(r):=\mathbb{y}\left(\mathbb{r}^{-1}(r)\right)$, we find

$$
\mathrm{r}^{-1}(r)=\int_{0}^{r}\left(w^{\prime}(z)+\frac{\Phi(w(z))}{\Phi^{\prime}(w(z))}\right) \mathrm{d} z
$$

Hence, by the reparametrization $\mathbb{y}(\theta)=w(\mathbb{r}(\theta))$, finding a maximizing function $\mathbb{y}$ for (4.9) reduces to the problem of finding a function $w$ which maximizes

$$
\begin{gathered}
J(w):=\int_{0}^{R} f(w(r)) e^{-(R-r)}\left(w^{\prime}(r)+\frac{\Phi(w(r))}{\Phi^{\prime}(w(r))}\right) \mathrm{d} r \quad\left(=\mathbb{E}\left[L_{\infty}\right]\right) \\
\text { subject to the condition } K(w):=\int_{0}^{R}\left(w^{\prime}(r)+\frac{\Phi(w(r))}{\Phi^{\prime}(w(r))}\right) \mathrm{d} r=\Theta_{0} .
\end{gathered}
$$

\section{Solving the calculus of variations problem}

In this section, we solve (locally) the calculus of variations problem of maximizing 4.10 subject to 4.11) by employing necessary and sufficient conditions on the first and second variation of the functionals involved. We obtain the candidate free boundary function $\mathrm{y}(\theta)$, see equations $(5.6)$ and (5.7), and show its local optimality in Lemma 5.4 We then relate our results on the calculus of variations problem to the initial optimal execution problem in Theorem 5.6. This will be crucial later for Section 6 to verify the desired inequality in the sell region, presented in Lemma 6.7

A maximizer $w$ of the isoperimetric problem $4.10-4.11$ also maximizes $J+m K$ for some constant $m:=m(R)$ that is the Lagrange multiplier, cf. GF00, Theorem 2.12.1]. Considering perturbations $w(r)+h(r)$ of $w$ with $h(0)=h(R)=0$, a necessary condition for an extremum $w$ of a functional $J+m K$ is that its first variation $\mathrm{D}(J+m K)$ vanishes at $w$, see GF00, Thm. 1.3.2]. Integration by parts yields the Euler-Lagrange equation

$$
0=F_{w}-\frac{\mathrm{d}}{\mathrm{d} r} F_{w^{\prime}}+\left(G_{w}-\frac{\mathrm{d}}{\mathrm{d} r} G_{w^{\prime}}\right) m,
$$

with $G\left(r, w, w^{\prime}\right):=w^{\prime}+\Phi(w) / \Phi^{\prime}(w)$ and $F\left(r, w, w^{\prime}\right):=f(w) e^{-(R-r)} G\left(r, w, w^{\prime}\right)$, the integrands of $K$ and $J$, respectively.

Since we assumed to start on the (yet unknown) boundary, one side is fixed, $w(R)=\mathrm{y}\left(\Theta_{0}\right)$. But the other end $w(0)$ is free. Thus, integration by parts of $\mathrm{D}(J+m K)$ with perturbations $w(r)+h(r)$ of $w$ where $h(0) \neq 0$ imposes as an additional condition for $\mathrm{D}(J+m K)$ to vanish that

$$
0=\left.\left(F_{w^{\prime}}+m G_{w^{\prime}}\right)\right|_{r=0} .
$$


This natural boundary condition (cf. GF00, Sect. 1.6]) yields the Lagrange multiplier $m(R)=-f\left(y_{0}\right) e^{-R}$ for $y_{0}:=\mathrm{y}(0)=w(0)$. After multiplication with $e^{R} \Phi^{\prime}(w)^{2}$, equation (5.1) simplifies to

$$
e^{r} \Phi(w)\left(f^{\prime}(w) \Phi^{\prime}(w)-f(w) \Phi^{\prime \prime}(w)\right)=f\left(y_{0}\right)\left(\Phi^{\prime}(w)^{2}-\Phi(w) \Phi^{\prime \prime}(w)\right) .
$$

Inserting $r=0$ gives a condition for $y_{0}$, namely

$$
f^{\prime}\left(y_{0}\right) \Phi\left(y_{0}\right)=f\left(y_{0}\right) \Phi^{\prime}\left(y_{0}\right) .
$$

Assumption C6 guarantees existence and C2 uniqueness of $y_{0}$. On the other hand, differentiating both sides of (5.2) with respect to $r$ gives the ODE for $w$

$$
\begin{aligned}
0= & \left(e^{r}\left(f^{\prime} \Phi^{\prime}-f \Phi^{\prime \prime}\right) \Phi^{\prime}+e^{r}\left(f^{\prime \prime} \Phi^{\prime}-f \Phi^{\prime \prime \prime}\right) \Phi-f\left(y_{0}\right)\left(\Phi^{\prime} \Phi^{\prime \prime}-\Phi \Phi^{\prime \prime \prime}\right)\right) w^{\prime} \\
& +e^{r}\left(f^{\prime} \Phi^{\prime}-f \Phi^{\prime \prime}\right) \Phi,
\end{aligned}
$$

where $f=f(w(r)), f^{\prime}=f^{\prime}(w(r)), \Phi=\Phi(w(r))$, etc.

Both sides in the above equality (5.2) are negative on the boundary $w(r)$, due to

Lemma 5.1. The positive, increasing eigenfunctions $\Phi=\Phi_{\delta}$ corresponding to the eigenvalue $\delta>0$ of the generator of an Ornstein-Uhlenbeck process satisfy

$$
\left(\Phi^{(n)}(x)\right)^{2}<\Phi^{(n-1)}(x) \Phi^{(n+1)}(x)
$$

for all $x \in \mathbb{R}$ and $n \in \mathbb{N}$. In particular, $\left(\Phi^{\prime}\right)^{2}<\Phi \Phi^{\prime \prime}$. Moreover, for $n \in \mathbb{N}$

$$
\lim _{x \rightarrow-\infty} \Phi^{(n)}(x) / \Phi^{(n-1)}(x)=0 \quad \text { and } \quad \lim _{x \rightarrow+\infty} \Phi^{(n)}(x) / \Phi^{(n-1)}(x)=+\infty .
$$

Proof. Since $H_{\nu}^{\prime}(x)=2 \nu H_{\nu-1}(x)$ for complex $\nu$ (see [Leb72, eq. (10.4.4)]), equation (2.6) implies

$$
\Phi_{\delta}^{(n)} \Phi_{\delta}^{(n+2)}-\left(\Phi_{\delta}^{(n+1)}\right)^{2}=\left(\Phi_{\delta+n \beta} \Phi_{\delta+n \beta}^{\prime \prime}-\left(\Phi_{\delta+n \beta}^{\prime}\right)^{2}\right) \frac{2^{2 n}}{\hat{\sigma}^{2 n} \beta^{n}} \prod_{k=0}^{n}(\delta+k \beta)^{2},
$$

so it suffices to prove $\left(\Phi^{\prime}\right)^{2}<\Phi^{\prime \prime} \Phi$ for every $\delta, \beta, \sigma, \hat{\sigma}>0$ and $\rho \in[-1,1]$ in 2.6 . This is equivalent to showing $\left(H_{\nu}^{\prime}\right)^{2}<H_{\nu}^{\prime \prime} H_{\nu}$ for every $\nu<0$. Since $\Gamma(-\nu)>0$ and $H_{\nu}(x)=\Gamma(-\nu)^{-1} \int_{0}^{\infty} e^{-t^{2}-2 x t} t^{-\nu-1} \mathrm{~d} t$ for $\nu<0$ (cf. Leb72, eq. (10.5.2)]), the function $\varphi_{x}(t):=e^{-t^{2}-2 x t} t^{-\nu-1}$ is the density of an absolutely continuous finite measure $\mu$ on $[0, \infty)$. For the probability measure $\tilde{\mathbb{P}}[A]:=\mu([0, \infty))^{-1} \mu(A)$ consider two independent random variables $X, Y \sim \tilde{\mathbb{P}}$. By [Kle08, Thm. 6.28], we can exchange differentiation and integration (in the integral representation of $H_{\nu}$ above) to see that $H_{\nu}^{\prime \prime}(x) H_{\nu}(x)-H_{\nu}^{\prime}(x)^{2}=4 \tilde{\mathbb{E}}\left[X^{2}-X Y\right]$. Symmetry gives $2 \tilde{\mathbb{E}}\left[X^{2}-X Y\right]=\tilde{\mathbb{E}}\left[(X-Y)^{2}\right] \geq 0$. Since $X$ and $Y$ are independent with absolutely continuous distribution, Fubini's theorem yields $\tilde{\mathbb{P}}[X=Y]=0$, so $\tilde{\mathbb{E}}\left[(X-Y)^{2}\right]>0$.

The asymptotic behavior of $\Phi^{(n)} / \Phi^{(n-1)}$ follows from [Leb72, eq. (10.6.4)] in the case $x \rightarrow-\infty$ and from [Leb72, eq. (10.6.7)] in the case $x \rightarrow+\infty$. 
Now (5.2) gives a representation of $r$ given $y_{0}$ and $w$ as

$$
r=\log \frac{f\left(y_{0}\right)}{\Phi(w)}+\log \frac{\Phi^{\prime}(w)^{2}-\Phi(w) \Phi^{\prime \prime}(w)}{f^{\prime}(w) \Phi^{\prime}(w)-f(w) \Phi^{\prime \prime}(w)},
$$

which we can use to simplify the ODE (5.3) (assuming $w^{\prime} \neq 0$ everywhere) to

$$
\frac{1}{w^{\prime}}=-\frac{\Phi^{\prime}}{\Phi}+\frac{f \Phi^{\prime \prime \prime}-f^{\prime \prime} \Phi^{\prime}}{f^{\prime} \Phi^{\prime}-f \Phi^{\prime \prime}}+\frac{\Phi^{\prime} \Phi^{\prime \prime}-\Phi \Phi^{\prime \prime \prime}}{\left(\Phi^{\prime}\right)^{2}-\Phi \Phi^{\prime \prime}},
$$

reading the right hand side as a function of $w(r)$. With $\mathbb{y}(\theta)=w(\mathbb{r}(\theta))$ and $r:=\mathbb{r}(\theta)$, we get $\mathbb{y}^{\prime}(\theta)=w^{\prime}(r) \mathbb{r}^{\prime}(\theta)=w^{\prime}(r)\left(1-\mathbb{y}^{\prime}(\theta)\right) \Phi^{\prime}(\mathbb{y}(\theta)) / \Phi(\mathbb{y}(\theta))$, which simplifies to

$$
\begin{aligned}
& \mathbb{y}^{\prime}(\theta)=\frac{\Phi^{\prime}(\mathrm{y})}{\Phi^{\prime}(\mathrm{y})+\Phi(\mathrm{y}) / w^{\prime}(r)} \\
&=\frac{1}{\Phi} \frac{\left(\left(\Phi^{\prime}\right)^{2}-\Phi \Phi^{\prime \prime}\right)\left(f^{\prime} \Phi^{\prime}-f \Phi^{\prime \prime}\right)}{\left(\Phi \Phi^{\prime \prime}-\left(\Phi^{\prime}\right)^{2}\right) f^{\prime \prime}+\left(\Phi^{\prime} \Phi^{\prime \prime}-\Phi \Phi^{\prime \prime \prime}\right) f^{\prime}+\left(\Phi^{\prime} \Phi^{\prime \prime \prime}-\left(\Phi^{\prime \prime}\right)^{2}\right) f} \\
&=\frac{M_{2}(\mathrm{y}(\theta))}{M_{1}^{\prime}(\mathrm{y}(\theta))}, \\
& \text { where } \quad M_{1}:=\frac{f \Phi^{\prime}-f^{\prime} \Phi}{\left(\Phi^{\prime}\right)^{2}-\Phi \Phi^{\prime \prime}} \quad \text { and } \quad M_{2}:=\frac{f^{\prime} \Phi^{\prime}-f \Phi^{\prime \prime}}{\left(\Phi^{\prime}\right)^{2}-\Phi \Phi^{\prime \prime}} .
\end{aligned}
$$

By 5.2 and Lemma 5.1 we have $M_{2}(\mathrm{y}(\theta))>0$ for any $\theta$. We get $M_{1}^{\prime}(\mathbb{y}(\theta))<0$ by

Lemma 5.2. Under Assumption C2, $M_{1}^{\prime}(y)<0$ for all $y \in \mathbb{R}$.

Proof. Let $G:=\Phi^{\prime} / \Phi$ and $H:=\Phi^{\prime \prime} / \Phi^{\prime}$. We have $G, G^{\prime}, H, H^{\prime}>0$ and $G<H$ by Lemma 5.1. With $\lambda(y)=f^{\prime}(y) / f(y)>0$, thus $f^{\prime \prime} / f=\lambda^{\prime}+\lambda^{2}$, we get

$$
\left(G^{\prime}\right)^{2} \Phi M_{1}^{\prime} / f=\lambda^{\prime} G^{\prime}+\left(\lambda^{2}-\lambda H\right) G^{\prime}+\left(G^{2}-\lambda G\right) H^{\prime} .
$$

So $M_{1}^{\prime}(y)<0$ if and only if $\lambda^{\prime}(y) G^{\prime}(y)<q(\lambda(y))$ where the right-hand side is $q(\lambda):=(H-\lambda) \lambda G^{\prime}+(\lambda-G) G H^{\prime}$. The function $q$ is quadratic in $\lambda$ and takes its minimum in

$$
\lambda^{*}:=\frac{H G^{\prime}+G H^{\prime}}{2 G^{\prime}} \quad \text { with value } \quad q\left(\lambda^{*}\right)=\frac{\left(H G^{\prime}+G H^{\prime}\right)^{2}}{4 G^{\prime}}-G^{2} H^{\prime} .
$$

Note also, that $G^{\prime}=(H-G) G$. We find that

$$
\begin{aligned}
4 G^{\prime}\left(\lambda^{\prime} G^{\prime}-q(\lambda)\right) & \leq 4 G^{\prime}\left(\lambda^{\prime} G^{\prime}-q\left(\lambda^{*}\right)\right)<4 G^{\prime}\left(\left(G^{\prime}\right)^{2}-q\left(\lambda^{*}\right)\right) \\
& =4\left(G^{\prime}\right)^{3}-\left(G H^{\prime}+G^{\prime} H\right)^{2}+4 G^{\prime} G^{2} H^{\prime} \\
& =G^{2}\left(4 G(H-G)^{3}-\left(H^{\prime}+(H-G) H\right)^{2}+4(H-G) G H^{\prime}\right) \\
& =-G^{2}\left(H^{\prime}+H^{2}+2 G^{2}-3 G H\right)^{2} \leq 0
\end{aligned}
$$

using that $\lambda^{\prime}(y)<G^{\prime}(y), y \in \mathbb{R}$, by Assumption $\mathrm{C} 2$, So $M_{1}^{\prime}(y)<0$ for all $y \in \mathbb{R}$. 
Lemma 5.3. Let $f$ satisfy Assumptions C2, C3 and C6. Then there exists a unique solution $\theta \mapsto \mathrm{y}(\theta), \theta \in[0, \infty)$, of the $O D E$

$$
\mathrm{y}^{\prime}=M_{2}(\mathrm{y}) / M_{1}^{\prime}(\mathrm{y}), \quad \mathrm{y}(0)=y_{0},
$$

and $\mathrm{y}$ is strictly decreasing to $\lim _{\theta \rightarrow \infty} \mathrm{y}(\theta)=y_{\infty}$ (with $y_{0}$ and $y_{\infty}$ from Assumption C6).

Proof. Since $M_{2} / M_{1}^{\prime}$ is locally Lipschitz by $f \in C^{3}(\mathbb{R})$, there exists a unique maximal solution $\mathbb{y}:\left[0, \theta_{\max }\right) \rightarrow \mathbb{R}$ of (5.7). We have $M_{2}(\mathrm{y}(\theta))>0$ and $M_{1}^{\prime}<0$ by Lemma 5.2 , thus $\mathrm{y}^{\prime}<0$. Assume $\theta_{\max }<\infty$, which implies $\lim _{\theta \rightarrow \theta_{\max }} \mathbb{y}(\theta)=-\infty$. However, note that $\left\{(\theta, \mathbb{y}(\theta)): 0 \leq \theta<\theta_{\max }\right\}$ and $[0, \infty) \times\left\{y_{\infty}\right\}$ are trajectories of the two-dimensional autonomous dynamical system induced by the field $(\theta, y) \mapsto\left(1, M_{2}(y) / M_{1}^{\prime}(y)\right)$. Since trajectories of autonomous dynamical systems cannot cross, and $y_{\infty}<y_{0}$ by Lemma 5.1 . we must have $y_{\infty}<\mathrm{y}(\theta)$ for all $\theta \in\left[0, \theta_{\max }\right)$, which contradicts $\theta_{\max }<\infty$.

Moreover, $\mathrm{y}^{-1}(y)=\int_{y_{0}}^{y}\left(M_{1}^{\prime} / M_{2}\right)(x) \mathrm{d} x$ is finite for every $y \in\left(y_{\infty}, y_{0}\right]$. Since $\theta_{\max }=\infty$, it follows that $\mathrm{y}(\theta) \rightarrow y_{\infty}$ as $\theta \rightarrow \infty$.

By considering the first variation $\mathrm{D}(J+m K)$, we found a candidate boundary function $\mathrm{y}$ in terms of a possible extremum $w:[0, R] \rightarrow \mathbb{R}$ of $J+m K$. Calculating the second variation $\mathrm{D}^{2}(J+m K)$ at $w$, we find that $w$ is indeed a local maximizer.

Lemma 5.4. The functional $\hat{J}:=J+m K: C^{1}([0, R]) \rightarrow \mathbb{R}$ defined by 4.10$)-$ (4.11) with $m=-f\left(y_{0}\right) e^{-R}$ has a strict local maximizer $w(r)=\mathrm{y}\left(\mathrm{r}^{-1}(r)\right)$, with $\mathbb{y}$ solving (5.7), in the following sense. There exists $\varepsilon>0$ such that for all perturbations $0 \not \equiv h \in C^{1}([0, R])$ with endpoints $h(0)=h(R)=0$ and $\|h\|_{W^{1, \infty}}=\|h\|_{\infty} \vee\left\|h^{\prime}\right\|_{\infty}<\varepsilon$ it holds

$$
\hat{J}(w+h)<\hat{J}(w) .
$$

Proof. For a $C^{1}$-perturbation $h:[0, R] \rightarrow \mathbb{R}$ of $w$ with $h(0)=h(R)=0$ we have by GF00, Sect. 5.25, (10) and (11)]

$$
\mathrm{D}^{2}(J+m K)[w ; h]=\int_{0}^{R}\left(P h^{\prime}(r)^{2}+Q h(r)^{2}\right) \mathrm{d} r
$$

with $P=P\left(r, w(r), w^{\prime}(r)\right)$ and $Q=Q\left(r, w(r), w^{\prime}(r)\right)$ given by

$$
\begin{aligned}
P & =\frac{1}{2}\left(F_{w^{\prime} w^{\prime}}+m G_{w^{\prime} w^{\prime}}\right)=0 \\
Q & =\frac{1}{2}\left(F_{w w}+m G_{w w}-\frac{\mathrm{d}}{\mathrm{d} r}\left(F_{w w^{\prime}}+m G_{w w^{\prime}}\right)\right) \\
& =\frac{1}{2} e^{-(R-r)}\left(\frac{\Phi}{\Phi^{\prime}} f^{\prime \prime}+2\left(\frac{\Phi}{\Phi^{\prime}}\right)^{\prime} f^{\prime}+\left(\frac{\Phi}{\Phi^{\prime}}\right)^{\prime \prime} f-f^{\prime}\right)+\frac{1}{2}\left(\frac{\Phi}{\Phi^{\prime}}\right)^{\prime \prime} m,
\end{aligned}
$$

with $f, \Phi$ and their derivatives being evaluated at $w(r)$ when no argument is mentioned. 
Differentiating (5.1) with respect to $r$ yields

$$
\begin{aligned}
0= & \frac{\mathrm{d}}{\mathrm{d} r} e^{-(R-r)}\left(\frac{\Phi}{\Phi^{\prime}} f^{\prime}+\left(\frac{\Phi}{\Phi^{\prime}}\right)^{\prime} f-f\right)+m \frac{\mathrm{d}}{\mathrm{d} r}\left(\frac{\Phi}{\Phi^{\prime}}\right)^{\prime} \\
= & e^{-(R-r)}\left(\frac{\Phi}{\Phi^{\prime}} f^{\prime}+\left(\frac{\Phi}{\Phi^{\prime}}\right)^{\prime} f-f\right) \\
& \quad+e^{-(R-r)}\left(\frac{\Phi}{\Phi^{\prime}} f^{\prime \prime}+2\left(\frac{\Phi}{\Phi^{\prime}}\right)^{\prime} f^{\prime}+\left(\frac{\Phi}{\Phi^{\prime}}\right)^{\prime \prime} f-f^{\prime}\right) w^{\prime}+\left(\frac{\Phi}{\Phi^{\prime}}\right)^{\prime \prime} m w^{\prime} \\
= & e^{-(R-r)}\left(\frac{\Phi}{\Phi^{\prime}} f^{\prime}+\left(\frac{\Phi}{\Phi^{\prime}}\right)^{\prime} f-f\right)+2 Q w^{\prime} \\
= & e^{-(R-r)} \frac{\Phi}{\left(\Phi^{\prime}\right)^{2}}\left(f^{\prime} \Phi^{\prime}-f \Phi^{\prime \prime}\right)+2 Q w^{\prime} .
\end{aligned}
$$

By equation (5.2) and Lemma 5.1, the first summand in 5.8 is negative along $w(r)$. Since $w(r)=\mathbb{y}\left(\mathrm{r}^{-1}(r)\right)$ and $\mathbb{r}^{-1}$ is strictly increasing, we have $w^{\prime}<0$ by Lemma 5.3 . So $Q\left(r, w(r), w^{\prime}(r)\right)<-\kappa<0$ on $[0, R]$ by (5.8) for some constant $\kappa=\kappa_{R}$, giving that the second variation is negative definite at $w$, i.e. for $h \not \equiv 0$,

$$
\mathrm{D}^{2}(J+m K)[w ; h]=\int_{0}^{R} Q\left(r, w(r), w^{\prime}(r)\right) h(r)^{2} \mathrm{~d} r<-\kappa \int_{0}^{R} h(r)^{2} \mathrm{~d} r<0 .
$$

To shorten notation, let $\hat{F}:=F+m G$, so $\hat{J}:=J+m K=\int_{0}^{R} \hat{F} \mathrm{~d} r$. Unless the arguments are explicitly written, take $\hat{F}=\hat{F}\left(r, w(r), w^{\prime}(r)\right)$. Taylor's theorem gives $\hat{J}(w+h)-\hat{J}(w)=\mathrm{D} \hat{J}[w ; h]+\mathrm{D}^{2} \hat{J}[w ; h]+\mathcal{E}(h)$ with first variation $\mathrm{D} \hat{J}[w ; h]=0$ by (5.1), second variation $\mathrm{D}^{2} \hat{J}[w ; h]=\int_{0}^{R} Q h^{2} \mathrm{~d} r<0$ by (5.9) and remainder

$$
\mathcal{E}(h)=\int_{0}^{R}\left(\sum_{|\boldsymbol{\alpha}|=3} \partial^{\boldsymbol{\alpha}} \hat{F}\left(r, \boldsymbol{w}+\xi_{r} \boldsymbol{h}\right) \frac{\boldsymbol{h}^{\boldsymbol{\alpha}}}{\boldsymbol{\alpha} !}\right) \mathrm{d} r
$$

for some $\xi_{r} \in[0,1]$, with $\boldsymbol{w}=\left(w(r), w^{\prime}(r)\right)^{\top}, \boldsymbol{h}=\left(h(r), h^{\prime}(r)\right)^{\top}$ and multi-index $\boldsymbol{\alpha} \in \mathbb{N}_{0}^{2}$, considering $\hat{F}(r, \cdot)$ as an function on $\mathbb{R}^{2}$. Since $\hat{F}$ is affine in $w^{\prime}$ we get

$$
\mathcal{E}(h)=\int_{0}^{R}\left(\frac{1}{6} \hat{F}_{w w w}\left(r, \boldsymbol{w}+\xi_{r} \boldsymbol{h}\right) h+\frac{1}{2} \hat{F}_{w w w^{\prime}}\left(r, \boldsymbol{w}+\xi_{r} \boldsymbol{h}\right) h^{\prime}\right) h^{2} \mathrm{~d} r=: \int_{0}^{R} A h^{2} \mathrm{~d} r
$$

Note that by compactness of $[0, R]$ we have uniform convergence

$$
\sup _{r \in[0, R]} \sup _{\xi \in[0,1]}\left|A\left(h(r), h^{\prime}(r), w(r), w^{\prime}(r), \xi, r\right)\right| \rightarrow 0
$$

as $\|h\|_{W^{1, \infty}} \rightarrow 0$. Now choose $\varepsilon>0$ small enough such that

$$
\left|A\left(h(r), h^{\prime}(r), w(r), w^{\prime}(r), \xi, r\right)\right|<\kappa / 2
$$

for all $r \in[0, R], \xi \in[0,1]$ and $h$ with $\|h\|_{W^{1, \infty}}<\varepsilon$. Hence, with $h \not \equiv 0$

$$
\hat{J}(w+h)-\hat{J}(w)=\int_{0}^{R}(Q+A) h^{2} \mathrm{~d} r<-\frac{\kappa}{2} \int_{0}^{R} h^{2} \mathrm{~d} r<0 .
$$


Note that the definition $w(r):=\mathrm{y}\left(\mathrm{r}^{-1}(r)\right)$ does not depend on the interval boundary $R$. Hence the optimizer $w$ over $[0, R]$ from Lemma 5.4 is optimal for all $R>0$. We can calculate the value $J(w)$ of our optimizer explicitly.

Lemma 5.5. For the optimal w from Lemma 5.4 we have

$$
J(w)=\left(\Phi M_{1}\right)\left(\mathbb{y}\left(\Theta_{0}\right)\right)=\left(\Phi M_{1}\right)(w(R)) .
$$

Proof. By direct calculation we have $f M_{1}^{\prime} /\left(\Phi M_{2}^{2}\right)=\left(\left(f \Phi^{\prime}-f^{\prime} \Phi\right) /\left(f^{\prime} \Phi^{\prime}-f \Phi^{\prime \prime}\right)\right)^{\prime}$. Moreover, (5.2) gives $e^{r}=f\left(y_{0}\right) /\left(\Phi M_{2}\right)(w(r))$. With $r=\mathbb{r}(\ell)$ and using (5.7), we get from (4.9) that

$$
\begin{aligned}
J(w) & =e^{-\mathrm{r}\left(\Theta_{0}\right)} \int_{0}^{\Theta_{0}} f(\mathbb{y}(\ell)) e^{\mathrm{r}(\ell)} \mathrm{d} \ell \\
& =\left(\Phi M_{2}\right)\left(\mathbb{y}\left(\Theta_{0}\right)\right) \int_{0}^{\Theta_{0}}\left(\frac{f}{\Phi M_{2}}\right)(\mathbb{y}(\ell)) \mathrm{d} \ell \\
& =\left(\Phi M_{2}\right)\left(\mathbb{y}\left(\Theta_{0}\right)\right) \int_{y_{0}}^{\mathbb{y}\left(\Theta_{0}\right)}\left(\frac{f M_{1}^{\prime}}{\Phi M_{2}^{2}}\right)(x) \mathrm{d} x \\
& =\left(\Phi M_{2}\right)\left(\mathbb{y}\left(\Theta_{0}\right)\right)\left[\frac{f \Phi^{\prime}-f^{\prime} \Phi}{f^{\prime} \Phi^{\prime}-f \Phi^{\prime \prime}}\right]_{y_{0}}^{\mathbb{y}\left(\Theta_{0}\right)} \\
& =\left(\Phi M_{1}\right)\left(\mathbb{y}\left(\Theta_{0}\right)\right) .
\end{aligned}
$$

Now we can translate the results obtained so far back to the state space of impact and asset position. The following theorem will be crucial for our analysis in the verification arguments in Section 6 .

Theorem 5.6. The function $\mathrm{y}:[0, \infty) \rightarrow \mathbb{R}$ defined by equation (5.7) is a (one-sided) local maximizer of $\mathbb{E}\left[L_{\infty}\left(A^{\text {refl }}\left(\mathbb{y}, \Theta_{0}\right)\right)\right]$ in the sense that, for every $\theta>0$ there exists $\varepsilon>0$ such that for any decreasing $\tilde{\mathrm{y}} \in C^{1}([0, \infty))$ with $\mathbb{y}(\cdot) \leq \tilde{\mathbb{y}}(\cdot) \leq y_{0}, \mathbb{y}=\tilde{\mathbb{y}}$ on $[\theta, \infty)$ and $0<\|\mathbb{y}-\tilde{y}\|_{W^{1, \infty}}<\varepsilon$, it holds

$$
\mathbb{E}\left[L_{\infty}\left(A^{\text {refl }}(\mathbb{y}, \theta)\right)\right]>\mathbb{E}\left[L_{\infty}\left(A^{\text {refl }}(\tilde{\mathrm{y}}, \theta)\right)\right] .
$$

Proof. For sake of clarity, we write $J=J_{R}$ and $K=K_{R}$ to emphasize the dependence of the functionals $J, K$ on $R$. Call $w(r)$ the parametrization of $\mathbb{y}$ and $\tilde{w}(r)$ the parametrization of $\tilde{\mathrm{y}}$.

Fix $\theta>0$ and choose $R, \hat{R}, \hat{\theta}$ such that $\mathrm{y}(\theta)=w(R), \tilde{\mathrm{y}}(\theta)=\tilde{w}(\hat{R})$ and $w(\hat{R})=\mathrm{y}(\hat{\theta})$. So $R:=\mathbb{r}_{\mathbf{y}}(\theta), \hat{R}:=\mathbb{r}_{\tilde{\mathbf{y}}}(\theta)=\int_{0}^{\theta} \frac{\Phi^{\prime}}{\Phi}(\tilde{\mathbb{y}}(x)) \mathrm{d} x+\int_{\tilde{\mathbf{y}}(\theta)}^{\tilde{\tilde{y}}(0)} \frac{\Phi^{\prime}}{\Phi}(u) \mathrm{d} u$ and $\hat{\theta}:=\mathbb{r}_{\mathbf{y}}^{-1}(\hat{R})$. By $\mathbb{y} \not \equiv \tilde{y}, \mathbb{y}(\cdot) \leq \tilde{\mathbb{y}}(\cdot)$ with equality outside $(0, \theta)$ and monotonicity of $\Phi^{\prime} / \Phi$, we have $\hat{R}>R$ and thus $\hat{\theta}>\theta$.

Now, $K_{\hat{R}}(w)=\hat{\theta}$ and $K_{\hat{R}}(\tilde{w})=\theta$. Moreover, $J_{r}(w)=\left(\Phi M_{1}\right)(w(r))$ by Lemma 5.5 . 
So if $\|w-\tilde{w}\|_{W^{1, \infty}}$ is small enough, by Lemma 5.4 we get

$$
\begin{aligned}
J_{R}(w) & =\left(\Phi M_{1}\right)(w(R))-\left(\Phi M_{1}\right)(w(\hat{R}))+J_{\hat{R}}(w) \\
= & \left(\Phi M_{1}\right)(w(R))-\left(\Phi M_{1}\right)(w(\hat{R}))+e^{-\hat{R}} f\left(y_{0}\right) \hat{\theta}+\left(J_{\hat{R}}-e^{-\hat{R}} f\left(y_{0}\right) K_{\hat{R}}\right)(w) \\
> & \left(\Phi M_{1}\right)(w(R))-\left(\Phi M_{1}\right)(w(\hat{R}))+e^{-\hat{R}} f\left(y_{0}\right) \hat{\theta}+\left(J_{\hat{R}}-e^{-\hat{R}} f\left(y_{0}\right) K_{\hat{R}}\right)(\tilde{w}) \\
= & \left(\Phi M_{1}\right)(\mathbb{y}(\hat{\theta}-\eta))-\left(\Phi M_{1}\right)(\mathbb{y}(\hat{\theta}))+e^{-\hat{R}} f\left(y_{0}\right) \eta+J_{\hat{R}}(\tilde{w}) \\
= & : \Psi(\eta)+J_{\hat{R}}(\tilde{w}) .
\end{aligned}
$$

where $\eta:=\hat{\theta}-\theta>0$. By (5.4) we get $e^{-\hat{R}} f\left(y_{0}\right)=\left(\Phi M_{2}\right)(\mathrm{y}(\hat{\theta}))$. With 5.5 follows

$$
\begin{aligned}
\Psi^{\prime}(\eta) & =-\left(\left(\Phi M_{1}\right)^{\prime} \frac{M_{2}}{M_{1}^{\prime}}\right)(\mathrm{y}(\hat{\theta}-\eta))+\left(\Phi M_{2}\right)(\mathrm{y}(\hat{\theta})) \\
& =-\left(\frac{\Phi^{\prime} M_{1} M_{2}}{M_{1}^{\prime}}+\Phi M_{2}\right)(\mathrm{y}(\hat{\theta}-\eta))+\left(\Phi M_{2}\right)(\mathrm{y}(\hat{\theta})) .
\end{aligned}
$$

Hence $\Psi^{\prime}(0)=-\left(\Phi^{\prime} M_{1} M_{2} / M_{1}^{\prime}\right)(\mathrm{y}(\hat{\theta}))$. Since $M_{1}>0$ on $\left(-\infty, y_{0}\right), M_{2}>0$ on $\left(y_{\infty}, y_{0}\right]$, $M_{1}^{\prime}<0$ by Lemma 5.2 and $\Phi^{\prime}>0$, it follows $\Psi^{\prime}(0)>0$. So $\Psi(\eta)>0$ for $\eta>0$ small enough. Therefore we have by 4.10

$$
\mathbb{E}\left[L_{\infty}\left(A^{\mathrm{refl}}(\mathrm{y}, \theta)\right)\right]=J_{R}(w)>J_{\hat{R}}(\tilde{w})=\mathbb{E}\left[L_{\infty}\left(A^{\mathrm{refl}}(\tilde{\mathrm{y}}, \theta)\right)\right] .
$$

The bounds on $\eta$ and $\|w-\tilde{w}\|_{W^{1, \infty}}$ are satisfied for small enough $\varepsilon>0$, because $(\mathrm{y}, \ell) \mapsto \mathbb{r}_{\mathrm{y}}(\ell)$ and $(\mathrm{y}, \ell) \mapsto \mathbb{r}_{\mathrm{y}}^{-1}(\ell)$ are continuous in $W^{1, \infty} \times \mathbb{R}$, so $\|w-\tilde{w}\|_{W^{1, \infty}} \rightarrow 0$, $\hat{R} \rightarrow R$ and $\hat{\theta} \rightarrow \theta$ as $\varepsilon \rightarrow 0$.

\section{Constructing the value function and verification}

In this section, we construct a candidate for the value function and verify the variational inequality (3.3) in Lemmas 6.6 and 6.7, relying on results from the previous sections. This will be sufficient to conclude the proof of our main result, Theorem 3.1

Having defined a candidate boundary via the ODE (5.7) to separate the sell and wait regions $\mathcal{S}$ and $\mathcal{W}$, we will now construct a solution $V$ of the variational inequality (3.3) that will give the value function of the optimal liquidation problem. As a direct consequence of Lemma 5.5, we get its value along the boundary

$$
V_{\text {bdry }}(\theta):=V(\mathbb{y}(\theta), \theta)=\Phi(\mathbb{y}(\theta)) M_{1}(y(\theta)) .
$$

Inside the wait region $\mathcal{W}$, which we assume is to the left of the boundary, we require $V=V^{\mathcal{W}}$ to satisfy $\frac{\hat{\sigma}^{2}}{2} V_{y y}+(\sigma \rho \hat{\sigma}-\beta y) V_{y}=\delta V$. Note that $V^{\mathcal{W}}$ solves the same ODE in $y$ as $\Phi$. Since $V$ should be also monotonically increasing, the only possibility is that $V^{\mathcal{W}}(y, \theta)=C(\theta) \Phi(y)$ for some increasing function $C:[0, \infty) \rightarrow[0, \infty)$. Using the boundary condition $V^{\mathcal{W}}(\mathrm{y}(\theta), \theta)=V_{\mathrm{bdry}}(\theta)$, in light of equation 6.1$)$ we then have

$$
V^{\mathcal{W}}(y, \theta):=\Phi(y) C(\theta)
$$


for $y \leq \mathbb{y}(\theta)$ and $\theta \geq 0$, where $C(\theta):=M_{1}(\mathbb{y}(\theta))$. On the other hand, in the sell region we require for $V=V^{\mathcal{S}}$ to satisfy $f=V_{y}^{\mathcal{S}}+V_{\theta}^{\mathcal{S}}$. We divide $\mathcal{S}$ in two parts:

$$
\begin{aligned}
& \mathcal{S}_{1}:=\left\{(y, \theta) \in \mathbb{R} \times(0, \infty): \mathbb{y}(\theta)<y<y_{0}+\theta\right\}, \\
& \mathcal{S}_{2}:=\left\{(y, \theta) \in \mathbb{R} \times(0, \infty): y_{0}+\theta<y\right\} .
\end{aligned}
$$

Let $\Delta:=\Delta(y, \theta) \geq 0$ denote the $\|\cdot\|_{\infty}$-distance of a point $(y, \theta) \in \overline{\mathcal{S}}$ to the boundary $\partial \mathcal{S}$ in direction $(-1,-1)$. This means in $\overline{\mathcal{S}}_{1}$ (but not in $\mathcal{S}_{2}$ ) that

$$
\mathbb{y}(\theta-\Delta)=y-\Delta \text {. }
$$

Inside $\overline{\mathcal{S}}_{1}$, we need to have

$$
V^{\mathcal{S}_{1}}(y, \theta):=V^{\mathcal{W}}(y-\Delta, \theta-\Delta)+\int_{y-\Delta}^{y} f(x) \mathrm{d} x,
$$

since $V_{y}^{\mathcal{S}_{1}}+V_{\theta}^{\mathcal{S}_{1}}=f$ in $\overline{\mathcal{S}}$ and $V^{\mathcal{S}_{1}}(\mathrm{y}(\theta), \theta)=V^{\mathcal{W}}(\mathrm{y}(\theta), \theta)$. Similarly, in $\overline{\mathcal{S}}_{2}$,

$$
V^{\mathcal{S}_{2}}(y, \theta):=\int_{y-\theta}^{y} f(x) \mathrm{d} x .
$$

To wrap up, the candidate value function is defined by:

$$
V=V^{\mathcal{W}} \text { on } \overline{\mathcal{W}}, \quad V=V^{\mathcal{S}_{1}} \text { on } \overline{\mathcal{S}}_{1}, \quad V=V^{\mathcal{S}_{2}} \text { on } \overline{\mathcal{S}}_{2} .
$$

The rest of this section is devoted to verifying that $V$ is a classical solution of the HJB variational inequality $(3.3)$ and thus concluding the proof of Theorem 3.1 by an application of the martingale optimality principle. We first formalize the heuristic verification from Section 3

\subsection{Martingale optimality principle}

Recall that $v$ is the value function of the optimal liquidation problem (cf. (2.5)).

Proposition 6.1 (Martingale optimality principle). Consider a $C^{2,1}$ function $V: \mathbb{R} \times[0, \infty) \rightarrow[0, \infty)$ with the following properties:

1. For every $\Theta_{0-} \geq 0$, there exist constants $C_{1}, C_{2}$ so that

$$
V(y, \theta) \leq C_{1} \exp \left(C_{2} y\right) \vee 1 \quad \text { for all }(y, \theta) \in \mathbb{R} \times\left[0, \Theta_{0-}\right] ;
$$

2. For every $\Theta_{0-} \geq 0$ and $A \in \mathcal{A}\left(\Theta_{0-}\right)$, the process $G$ from (3.1) is a supermartingale, where $Y=Y^{y, A}$ is defined in (2.3), and additionally $G_{0}(y ; A) \leq G_{0-}(y ; A)$.

Then we have $\bar{S}_{0} \cdot V(y, \theta) \geq v(y, \theta)$.

Moreover, if there exists $A^{*} \in \mathcal{A}\left(\Theta_{0_{-}}\right)$such that $G\left(y ; A^{*}\right)$ is a martingale and $G_{0}\left(y ; A^{*}\right)=G_{0-}\left(y ; A^{*}\right)$ holds, then we have $\bar{S}_{0} V(y, \theta)=v(y, \theta)$ and $v(y, \theta)=\mathbb{E}\left[L_{\infty}\left(y ; A^{*}\right)\right]$ for $\Theta_{0-}=\theta \geq 0$. In this case, any strategy $A$ for which $G(y ; A)$ is not a martingale would be suboptimal. 
Proof. By the supermartingale property we have for every $T \geq 0$

$$
\begin{aligned}
\bar{S}_{0} V\left(Y_{0-}, \Theta_{0-}\right) & \geq \mathbb{E}\left[G_{0}(y ; A)\right] \geq \mathbb{E}\left[L_{T}(y ; A)+e^{-\gamma T} \bar{S}_{T} V\left(Y_{T}, \Theta_{T}\right)\right] \\
& =\mathbb{E}\left[L_{T}(y ; A)\right]+e^{-\gamma T} \mathbb{E}\left[\bar{S}_{T} V\left(Y_{T}, \Theta_{T}\right)\right] \\
& =\mathbb{E}\left[L_{T}(y ; A)\right]+e^{-\delta T} \bar{S}_{0} \mathbb{E}\left[\mathcal{E}(\sigma W)_{T} V\left(Y_{T}, \Theta_{T}\right)\right] .
\end{aligned}
$$

By monotone convergence, the first summand in $(6.7)$ tends to $\mathbb{E}\left[L_{\infty}(y ; A)\right]$ for $T \rightarrow \infty$. To see that the second summand converges to 0 , consider the Ornstein-Uhlenbeck process $\mathrm{d} X_{t}=-\beta X_{t} \mathrm{~d} t+\hat{\sigma} \mathrm{d} B_{t}, X_{0}=y$. An application of Itô's formula gives

$$
e^{\beta t}\left(Y_{t}-X_{t}\right)=\int_{[0, t]} e^{\beta u} \mathrm{~d} \Theta_{u} \quad \forall t \geq 0 .
$$

Since $\Theta$ is non-increasing, we conclude $Y_{t} \leq X_{t}$ for all $t \geq 0$. Let $p, q>1$ be conjugate, i.e. $1=1 / q+1 / p$. Using Hölder's inequality and the bound on $V$,

$$
\begin{aligned}
\mathbb{E}\left[\mathcal{E}(\sigma W)_{T} V\left(Y_{T}, \Theta_{T}\right)\right] \leq \mathbb{E}\left[\mathcal{E}(\sigma W)_{T}^{p}\right]^{1 / p} \mathbb{E}\left[V\left(Y_{T}, \Theta_{T}\right)^{q}\right]^{1 / q} \\
\quad=\mathbb{E}\left[\exp \left(p \sigma W_{T}-\frac{1}{2} p \sigma^{2} T\right)\right]^{1 / p} \mathbb{E}\left[V\left(Y_{T}, \Theta_{T}\right)^{q}\right]^{1 / q} \\
\quad=\mathbb{E}\left[\mathcal{E}(p \sigma W)_{T}\right]^{1 / p} \exp \left(\frac{1}{p}\left(\frac{1}{2} p^{2} \sigma^{2} T-\frac{1}{2} p \sigma^{2} T\right)\right) \mathbb{E}\left[V\left(Y_{T}, \Theta_{T}\right)^{q}\right]^{1 / q} \\
\quad=\exp \left(\frac{p-1}{2} \sigma^{2} T\right) \mathbb{E}\left[V\left(Y_{T}, \Theta_{T}\right)^{q}\right]^{1 / q} \\
\quad \leq \exp \left(\frac{p-1}{2} \sigma^{2} T\right) \mathbb{E}\left[C_{1}^{q} \exp \left(q C_{2} Y_{T}\right) \vee 1\right]^{1 / q} \\
\quad \leq \exp \left(\frac{p-1}{2} \sigma^{2} T\right) \mathbb{E}\left[C_{1}^{q} \exp \left(q C_{2} X_{T}\right) \vee 1\right]^{1 / q} .
\end{aligned}
$$

Using the fact that $X$ is a Gaussian process with mean $\mathbb{E}\left[X_{T}\right]=y e^{-\beta T}$ and variance $\operatorname{Var}\left(X_{T}\right)=\frac{\hat{\sigma}^{2}}{2 \beta}\left(1-e^{-2 \beta T}\right)$, we get for $K:=\mathbb{E}\left[C_{1}^{q} \exp \left(q C_{2} X_{T}\right) \vee 1\right]$ that

$$
\begin{aligned}
K & \leq 1+C_{1}^{q} \exp \left(q C_{2} \mathbb{E}\left[X_{T}\right]+\frac{1}{2} q^{2} C_{2}^{2} \operatorname{Var}\left(X_{T}\right)\right) \\
& \leq 1+C_{1}^{q} \exp \left(q C_{2} y+\frac{\hat{\sigma}^{2}}{4 \beta} q^{2} C_{2}^{2}\right) .
\end{aligned}
$$

This bound on $K$ is independent of $T$. Now choosing $p>1$ such that $\frac{p-1}{2} \sigma^{2}<\delta$ ensures that $\exp (-\delta T) \exp \left(\frac{p-1}{2} \sigma^{2} T\right)$ is exponentially decreasing in $T$, and thus the second summand in (6.7) converges to 0 for $T \rightarrow \infty$. This implies that $\bar{S}_{0} V(y, \theta) \geq \mathbb{E}\left[L_{\infty}(y ; A)\right]$ for all $A \in \mathcal{A}(\theta)$ and yields the first part of the claim. The second part follows similarly by noting that, if $A^{*} \in \mathcal{A}(\theta)$ is such that $G\left(y ; A^{*}\right)$ is a martingale and $G_{0}(y ; A)=G_{0-}(y ; A)$, then we have equalities instead of inequalities in the estimates leading to 6.7). By taking $T \rightarrow \infty$ we conclude that $\bar{S}_{0} V(y, \theta)=\mathbb{E}\left[L_{\infty}\left(y ; A^{*}\right)\right]$. Since $\bar{S}_{0} V(y, \theta) \geq v(y, \theta)$ by the first part of the claim, we deduce the optimality of $A^{*}$.

To justify later why the stochastic integrals in $(3.2)$ are true martingales, we need the following technical 
Lemma 6.2. Let $\Theta_{0-} \geq 0$ be given and $F \in C^{2,1}(\mathbb{R} \times[0, \infty) ; \mathbb{R})$ be such that there exist constants $C_{1}, C_{2} \geq 0$ with $|F(y, \theta)| \leq C_{1} \exp \left(C_{2} y\right) \vee 1$ for all $(y, \theta) \in \mathbb{R} \times\left[0, \Theta_{0-}\right]$. For an admissible strategy $A \in \mathcal{A}\left(\Theta_{0-}\right)$ let $Y^{A}=: Y$ denote the impact process defined by (2.3) for $y \in \mathbb{R}$. Then the stochastic integral processes

$$
\int_{0}^{\cdot} \bar{S}_{u} F\left(Y_{u}, \Theta_{u}\right) \mathrm{d} B_{u} \quad \text { and } \quad \int_{0}^{\cdot} \bar{S}_{u} F\left(Y_{u}, \Theta_{u}\right) \mathrm{d} W_{u} \quad \text { are true martingales. }
$$

Proof. It suffices to check $\mathbb{E}\left[\int_{0}^{t} \bar{S}_{u}^{2} \exp \left(2 C_{2} Y_{u}\right) \mathrm{d} u\right]<\infty$ for every $t \geq 0$ by the exponential growth of $F$. Consider the Ornstein-Uhlenbeck process $X$ given by $\mathrm{d} X_{t}=-\beta X_{t} \mathrm{~d} t+\hat{\sigma} \mathrm{d} B_{t}$, with $X_{0}=y$. As in the proof of Proposition 6.1 (see (6.8), we have $Y_{t} \leq X_{t}$ for all $t \geq 0$. In particular,

$$
\begin{aligned}
& \mathbb{E}\left[\int_{0}^{t} \bar{S}_{u}^{2} \exp \left(2 C_{2} Y_{u}\right) \mathrm{d} u\right] \leq \mathbb{E}\left[\int_{0}^{t} \bar{S}_{u}^{2} \exp \left(2 C_{2} X_{u}\right) \mathrm{d} u\right] \\
& =\int_{0}^{t} \mathbb{E}\left[\bar{S}_{u}^{2} \exp \left(2 C_{2} X_{u}\right)\right] \mathrm{d} u \leq \int_{0}^{t} \sqrt{\mathbb{E}\left[\bar{S}_{u}^{4}\right] \mathbb{E}\left[\exp \left(4 C_{2} X_{u}\right)\right]} \mathrm{d} u<\infty,
\end{aligned}
$$

using the Cauchy-Schwarz inequality and the fact that $X$ is a Gaussian process.

\subsection{Verification and proof of Theorem 3.1}

Now we verify that $V$ is a classical solution of the variation inequality 3.3 with the boundary condition $V(y, 0)=0$ for all $y \in \mathbb{R}$. That $V(y, 0)=0$ is clear because $M_{1}\left(y_{0}\right)=0$. The rest will be split into several lemmas.

Lemma 6.3 (Smooth pasting). Let $\left(y_{b}, \theta_{b}\right) \in \overline{\mathcal{W}} \cap \overline{\mathcal{S}}$. Then

$$
\begin{aligned}
\Phi\left(y_{b}\right) C^{\prime}\left(\theta_{b}\right)+\Phi^{\prime}\left(y_{b}\right) C\left(\theta_{b}\right) & =f\left(y_{b}\right), \\
\Phi^{\prime}\left(y_{b}\right) C^{\prime}\left(\theta_{b}\right)+\Phi^{\prime \prime}\left(y_{b}\right) C\left(\theta_{b}\right) & =f^{\prime}\left(y_{b}\right) .
\end{aligned}
$$

Proof. This follows easily from $C\left(\theta_{b}\right)=M_{1}\left(y_{b}\right)$ and $C^{\prime}\left(\theta_{b}\right)=M_{2}\left(y_{b}\right)$, see the definition of $C$ and (5.7), together with the definitions of $M_{1}$ and $M_{2}$, see (5.6). Note that when $\left(y_{b}, \theta_{b}\right)=\left(y_{0}, 0\right)$ we take the right derivative of $C$ at 0 and the equalities still hold true.

Remark 6.4. It might be interesting to point out that $(6.9)$ and $(6.10)$ are sufficient to derive the boundary between the sell and the wait regions. Indeed, solving (6.9) - 6.10 with respect to $C\left(\theta_{b}\right)$ and $C^{\prime}\left(\theta_{b}\right)$, it is easy to see that $C\left(\theta_{b}\right)=M_{1}\left(y_{b}\right)$ and $C^{\prime}\left(\theta_{b}\right)=M_{2}\left(y_{b}\right)$. On the other hand, by the chain rule one gets $\theta^{\prime}\left(y_{b}\right) C^{\prime}\left(\theta_{b}\right)=M_{1}^{\prime}\left(y_{b}\right)$ and thus we derive for the boundary parametrization $\theta(\cdot)=\mathrm{y}^{-1}(\cdot)$ in the appropriate range

$$
\theta^{\prime}\left(y_{b}\right)=\frac{M_{1}^{\prime}}{M_{2}}\left(y_{b}\right)
$$

which gives the ODE for the boundary in (5.7). To get the initial condition $y_{0}$, note that the boundary condition $V(\cdot, 0) \equiv 0$ gives $C(0)=0$, i.e. $M_{1}\left(y_{0}\right)=0$, exactly as 
in Lemma 5.3. Thus, one could derive the candidate boundary function $\mathrm{y}(\cdot)$ after assuming sufficient smoothness of the function $V$ along the boundary. This is similar to the classical approach in the singular stochastic control literature, cf. [KS86, Section 6]. The reason why we chose the seemingly longer derivation via calculus of variation techniques is the local (one-sided) optimality that we derived in Theorem 5.6 and that will be crucial in our verification of the inequalities of the candidate value function in the sell region, see Lemma 6.7. Even in the special case of $\lambda(\cdot)$ being constant, a more direct approach to verify the variational inequality is suggesting new, yet unproven (to our best knowledge), properties for quotients of Hermite functions that might be of independent interest, see Remark 6.8 .

The smooth-pasting property translates to smoothness of $V$. Moreover, exponential bound on $V$ and $V_{y}$ will be needed to rely on the verification results from Section 6.1 .

Lemma 6.5. The function $V$ is $C^{2,1}(\mathbb{R} \times[0, \infty))$. Moreover, for every $\Theta_{0-}$ there exist constants $C_{1}, C_{2}$, that depend on $\Theta_{0-}$, such that both $V(y, \theta)$ and $V_{y}(y, \theta)$ are non-negative and bounded from above by $C_{1} \exp \left(C_{2} y\right) \vee 1$ for all $(y, \theta) \in \mathbb{R} \times\left[0, \Theta_{0-}\right]$.

Proof. In $\mathcal{W}$, the function $V$ is already $C^{2,1}$ by construction and the fact that $C(\theta)=M_{1}(\mathbb{y}(\theta))$ is continuously differentiable since $\mathbb{y}(\cdot)$ and $M_{1}(\cdot)$ are so.

For $(y, \theta) \in \mathcal{S}_{1}$, set $\left(y_{b}, \theta_{b}\right):=(y-\Delta(y, \theta), \theta-\Delta(y, \theta))$ and $\Delta:=\Delta(y, \theta)$ (recall (6.3). We have by (6.4) for the first and 6.9 for the second equality

$$
\begin{aligned}
V_{y}^{\mathcal{S}_{1}} & =\Phi^{\prime}\left(y_{b}\right) C\left(\theta_{b}\right)\left(1-\Delta_{y}\right)+\Phi\left(y_{b}\right) C^{\prime}\left(\theta_{b}\right)\left(-\Delta_{y}\right)+f(y)-f\left(y_{b}\right)\left(1-\Delta_{y}\right) \\
& =\Phi^{\prime}(y-\Delta) C(\theta-\Delta)+f(y)-f(y-\Delta)
\end{aligned}
$$

Since $f, \Delta, C$ and $\Phi^{\prime}$ are continuously differentiable, $V_{y}$ will also be so. Hence by 6.10 ,

$$
\begin{aligned}
V_{y y}^{\mathcal{S}_{1}} & =\Phi^{\prime \prime}\left(y_{b}\right) C\left(\theta_{b}\right)\left(1-\Delta_{y}\right)+\Phi^{\prime}\left(y_{b}\right) C^{\prime}\left(\theta_{b}\right)\left(-\Delta_{y}\right)+f^{\prime}(y)-f^{\prime}\left(y_{b}\right)\left(1-\Delta_{y}\right) \\
& =V_{y y}^{\mathcal{W}}\left(y_{b}, \theta_{b}\right)+f^{\prime}(y)-f^{\prime}\left(y_{b}\right),
\end{aligned}
$$

which is continuous. On the other hand, by 6.4 and 6.10 we have

$$
\begin{aligned}
V_{\theta}^{\mathcal{S}_{1}}(y, \theta) & =\Phi^{\prime}\left(y_{b}\right) C\left(\theta_{b}\right)\left(-\Delta_{\theta}\right)+\Phi\left(y_{b}\right) C^{\prime}\left(\theta_{b}\right)\left(1-\Delta_{\theta}\right)-f\left(y_{b}\right)\left(-\Delta_{\theta}\right) \\
& =\Phi\left(y_{b}\right) C^{\prime}\left(\theta_{b}\right)
\end{aligned}
$$

which is continuous. For $(y, \theta) \in \overline{\mathcal{W}} \cap \overline{\mathcal{S}}$ on the boundary, the left derivative w.r.t. $y$ is

$$
\lim _{x \searrow 0} \frac{1}{x}(V(y, \theta)-V(y-x, \theta))=\Phi(y) C(\theta),
$$

while the right derivative is again given by 6.11 and is equal to the left derivative since $\Delta(y, \theta)=0$ in this case. Hence, $V$ is continuously differentiable w.r.t. $y$ on the boundary with derivative $V_{y}(y, \theta)=\Phi^{\prime}(y) C(\theta)$. Similarly, the left derivative of $V_{y}$ on the boundary is $\Phi^{\prime \prime}(y) C(\theta)$ and is equal to the right derivative which is given by 6.12 
with $y=y_{b}$. The left derivative of $V$ w.r.t. $\theta$ on the boundary is equal to the right derivative (given by (6.13p). Therefore, $V$ is $C^{2,1}$ inside $\overline{\mathcal{W}} \cup \mathcal{S}_{1}$.

For $(y, \theta) \in \mathcal{S}_{2}$, we have that $V_{y}^{\mathcal{S}_{2}}=f(y)-f(y-\theta), V_{y y}^{\mathcal{S}_{2}}=f^{\prime}(y)-f^{\prime}(y-\theta)$ and $V_{\theta}^{\mathcal{S}_{2}}=f(y-\theta)$ by 6.5 , which are all continuous. On the boundary between $\mathcal{S}_{1}$ and $\mathcal{S}_{2}$, the left derivative of $V$ w.r.t. $y$ is given by 6.11) while the right derivative is $f(y)-f\left(y_{0}\right)$. Since $\theta-\Delta=0$ in this case and $C(0)=0$, they are equal and hence $V$ is continuously differentiable w.r.t. $y$ there; similarly for $V_{y y}$. The left derivative of $V$ w.r.t. $\theta$ there is given by $(6.13)$ with $\left(y_{b}, \theta_{b}\right)=\left(y_{0}, 0\right)$. The right derivative w.r.t. $\theta$ is $f(y-\theta)=f\left(y_{0}\right)$. They are equal by $(6.10)$ and $C(0)=0$. Therefore, $V$ is $C^{2,1}$ on $\overline{\mathcal{S}}_{1} \cup \mathcal{S}_{2}$. It remains to check smoothness on $\{(y, 0): y \in \mathbb{R}\}$. The derivatives w.r.t. $y$ there are $0 . V$ is continuously differentiable w.r.t. $\theta$ in this case because $\mathrm{y}(\cdot), C$, and $\Delta$ are continuously differentiable w.r.t. $\theta$ also at $\theta=0$ (we consider the right derivatives in this case).

To conclude the proof, the bound of $V$ and $V_{y}$ can be argued as follows. In the wait region, which is contained in $\left(-\infty, y_{0}\right] \times[0, \infty)$, we have $V(y, \theta)=C(\theta) \Phi(y)$ and $V_{y}(y, \theta)=C(\theta) \Phi^{\prime}(y)$. Since $\Phi, \Phi^{\prime}$ are strictly increasing in $y$ (see 2.6) and Leb72, Chapter 10] for properties of the Hermite functions), $V$ and $V_{y}$ will be bounded by a constant there. Now, in the sell region we have $f-V_{y}-V_{\theta}=0$. However, $V_{\theta}>0$ because in $\mathcal{S}_{1}(6.13)$ holds and $C^{\prime}\left(\theta_{b}\right)=M_{2}\left(\mathrm{y}\left(\theta_{b}\right)\right)>0$, while in $\mathcal{S}_{2}$ we have that $V_{\theta}(y, \theta)=f(y-\theta)>0$. Similarly, $V_{y}>0$ in the sell region. Therefore, $0<V_{y}(y, \theta)<f(y) \leq \exp \left(\lambda_{\infty} y\right) \vee 1$ by Assumption C4 Hence, integrating in $y$ gives $V(y, \theta) \leq V(0, \theta)+\exp \left(\lambda_{\infty} y\right) / \lambda_{\infty}$ for $y \geq 0$, which implies $V(y, \theta) \leq C_{1} \exp \left(C_{2} y\right) \vee 1$ for appropriate constants $C_{1}, C_{2}$.

Next we prove that $V$ solves the variational inequality (3.3).

Lemma 6.6. The function $V^{\mathcal{W}}: \overline{\mathcal{W}} \rightarrow[0, \infty)$ from $(6.2)$ satisfies

$$
\mathcal{L} V^{\mathcal{W}}(y, \theta)=0 \quad \text { and } \quad f(y)<V_{y}^{\mathcal{W}}(y, \theta)+V_{\theta}^{\mathcal{W}}(y, \theta) \text { for } y<y(\theta) .
$$

Proof. By [5.5), we have $V_{\theta}^{\mathcal{W}}=\Phi(y) M_{1}^{\prime}(\mathrm{y}(\theta)) \mathrm{y}^{\prime}(\theta)=\Phi(y) M_{2}(\mathrm{y}(\theta))$ and $V_{y}^{\mathcal{W}}=\Phi^{\prime}(y) M_{1}(\mathrm{y}(\theta))$. Recall that at $y=\mathrm{y}(\theta)$ we have by $(6.9)$ the equality $V_{y}^{\mathcal{W}}+V_{\theta}^{\mathcal{W}}=f(\mathrm{y}(\theta))$. Now consider $y<\mathrm{y}(\theta)$. By Lemma 5.2 , we then have $M_{1}(y)>M_{1}(\mathrm{y}(\theta))$ giving

$$
\left(\frac{f}{\Phi}\right)^{\prime}(y)>\left(\frac{\Phi^{\prime}}{\Phi}\right)^{\prime}(y) M_{1}(\mathrm{y}(\theta))=\frac{\mathrm{d}}{\mathrm{d} y}\left(M_{1}(\mathrm{y}(\theta)) \frac{\Phi^{\prime}(y)}{\Phi(y)}+M_{2}(\mathrm{y}(\theta))\right) .
$$

Therefore, $y \mapsto\left(f-V_{y}^{\mathcal{W}}(y, \theta)+V_{\theta}^{\mathcal{W}}(y, \theta)\right) / \Phi(y)$ is increasing in $y$. Since at $y=\mathrm{y}(\theta)$ it equals to 0 , we get the claimed inequality.

It remains to verify the inequality in the sell region. The proof is more subtle and that is where Theorem 5.6 plays a crucial role. Recall Assumption 2.2 and note that $y_{\infty}$ from Lemma 5.3 is unique by condition C3.

Lemma 6.7. The functions $V^{\mathcal{S}_{1}}$ and $V^{\mathcal{S}_{2}}$ satisfy on $\overline{\mathcal{S}}_{1}$ and $\mathcal{S}_{2}$ respectively

$$
\mathcal{L} V^{\mathcal{S}_{1}} \leq 0, \quad \mathcal{L} V^{\mathcal{S}_{2}}<0 .
$$


Moreover, the inequality inside $\overline{\mathcal{S}}_{1}$ is strict except on the boundary between the wait region and the sell region $\left(\overline{\mathcal{W}} \cap \overline{\mathcal{S}}_{1}\right)$ where we have equality.

Proof. First consider region $\overline{\mathcal{S}}_{1}$. Recall from Lemma 6.5 (see 6.11 - 6.12) that in this case

$$
\begin{aligned}
& V_{y}^{\mathcal{S}_{1}}(y, \theta)=V_{y}^{\mathcal{W}}(y-\Delta, \theta-\Delta)+f(y)-f(y-\Delta), \\
& V_{y y}^{\mathcal{S}_{1}}(y, \theta)=V_{y y}^{\mathcal{W}}\left(y_{b}, \theta_{b}\right)+f^{\prime}(y)-f^{\prime}\left(y_{b}\right),
\end{aligned}
$$

where $y=y_{b}+\Delta(y, \theta)$ and $\theta=\theta_{b}+\Delta(y, \theta)$. Fix $\left(y_{b}, \theta_{b}\right) \in \overline{\mathcal{W}} \cap \overline{\mathcal{S}}_{1}$ and consider the perturbation $\Delta \mapsto(y, \theta)=\left(y_{b}+\Delta, \theta_{b}+\Delta\right)$. Set

$$
\begin{aligned}
h(\Delta) & :=\mathcal{L} V^{\mathcal{S}_{1}}\left(y_{b}+\Delta, \theta_{b}+\Delta\right) \\
= & \frac{\hat{\sigma}^{2}}{2} V_{y y}^{\mathcal{W}}\left(y_{b}, \theta_{b}\right)-\frac{\hat{\sigma}^{2}}{2} f^{\prime}\left(y_{b}\right)+\sigma \rho \hat{\sigma} V_{y}^{\mathcal{W}}\left(y_{b}, \theta_{b}\right)-\sigma \rho \hat{\sigma} f\left(y_{b}\right)-\delta V^{\mathcal{W}}\left(y_{b}, \theta_{b}\right) \\
& +\frac{\hat{\sigma}^{2}}{2} f^{\prime}(y)-\beta y V_{y}^{\mathcal{W}}\left(y_{b}, \theta_{b}\right)+\beta y f\left(y_{b}\right)+(\sigma \rho \hat{\sigma}-\beta y) f(y)-\delta \int_{y_{b}}^{y} f(x) \mathrm{d} x .
\end{aligned}
$$

Note that $h(0)=0$ by Lemma 6.6 and to show $h(\Delta)<0$ for $\Delta>0$, it suffices to prove $h^{\prime}(\Delta)<0$ for all $\Delta>0$. We have for all $\Delta \geq 0$ at $y=y_{b}+\Delta$ that

$$
\begin{aligned}
h^{\prime}(\Delta)=\beta( & \left.f\left(y_{b}\right)-V_{y}^{\mathcal{W}}\left(y_{b}, \theta_{b}\right)\right) \\
+f(y) & (\underbrace{\frac{\hat{\sigma}^{2}}{2} \frac{f^{\prime \prime}(y)}{f(y)}-(\beta+\delta)+(\sigma \rho \hat{\sigma}-\beta y) \frac{f^{\prime}(y)}{f(y)}}_{=k(y)}),
\end{aligned}
$$

where at $\Delta=0$ we consider the right derivative $h^{\prime}(0+)$. Now we show that $k(y)<0$ for all $y \geq y_{\infty}$. To this end, recall that $\Phi$ is a solution of the $\operatorname{ODE} \delta \Phi(x)=\frac{\hat{\sigma}^{2}}{2} \Phi^{\prime \prime}(x)+(\sigma \rho \hat{\sigma}-\beta x) \Phi^{\prime}(x)$. Differentiating w.r.t. $x$ and dividing by $\Phi^{\prime}(x)$ yields

$$
0=\frac{\hat{\sigma}^{2}}{2}\left(\frac{\Phi^{\prime \prime}(x)}{\Phi^{\prime}(x)}\right)^{\prime}+\frac{\hat{\sigma}^{2}}{2} \frac{\Phi^{\prime \prime}(x)^{2}}{\Phi^{\prime}(x)^{2}}-(\beta+\delta)+(\sigma \rho \hat{\sigma}-\beta x) \frac{\Phi^{\prime \prime}(x)}{\Phi^{\prime}(x)}
$$

So at the left end $y_{\infty}$ of our boundary, we have

$$
\begin{aligned}
k\left(y_{\infty}\right) & =\frac{\hat{\sigma}^{2}}{2}\left(\frac{f^{\prime}}{f}\right)^{\prime}\left(y_{\infty}\right)+\frac{\hat{\sigma}^{2}}{2} \frac{\Phi^{\prime \prime}\left(y_{\infty}\right)^{2}}{\Phi^{\prime}\left(y_{\infty}\right)^{2}}-(\beta+\delta)+\left(\sigma \rho \hat{\sigma}-\beta y_{\infty}\right) \frac{\Phi^{\prime \prime}\left(y_{\infty}\right)}{\Phi^{\prime}\left(y_{\infty}\right)} \\
& =\frac{\hat{\sigma}^{2}}{2}\left(\frac{f^{\prime}}{f}\right)^{\prime}\left(y_{\infty}\right)-\frac{\hat{\sigma}^{2}}{2}\left(\frac{\Phi^{\prime \prime}}{\Phi^{\prime}}\right)^{\prime}\left(y_{\infty}\right)<0
\end{aligned}
$$

by Assumption C3. With Assumption C5 we get $k(y)<0$ for every $y \geq y_{\infty}$.

In particular, $k\left(y_{b}+\Delta\right)<0$ for all $\Delta \geq 0$. Since $f$ is positive and increasing, the product $\Delta \mapsto(f k)\left(y_{b}+\Delta\right)$ is decreasing. Therefore, proving $h^{\prime}(0+) \leq 0$ is sufficient to show the inequality in $\mathcal{S}_{1}$. To stress the dependence of $h$ on the point $\left(y_{b}, \theta_{b}\right)=\left(\mathbb{y}\left(\theta_{b}\right), \theta_{b}\right)$, we also write $h(\Delta)=h_{\theta_{b}}(\Delta)$. Note that $h_{\theta}(\Delta)$ is continuous in $\theta$ and $\Delta$ on $[0, \infty) \times[0, \infty)$. 
Assume $h_{\theta_{b}}^{\prime}(0+)>0$ at some boundary point $\left(y_{b}, \theta_{b}\right)$ with $\theta_{b}>0$. By continuity of $h^{\prime}$ on $\theta$ and $\Delta$ there exists some $\varepsilon>0$ such that $\mathcal{L} V^{\mathcal{S}_{1}}>0$ on $U:=\overline{\mathcal{S}}_{1} \cap B_{\varepsilon}\left(y_{b}, \theta_{b}\right)$. This will lead to a contradiction to the fact that the candidate boundary is a (one-sided) strict local maximizer of our stochastic optimization problem with strategies described by the local times of reflected diffusions, see Theorem 5.6 .

Indeed, fix $\Theta_{0}>\theta_{b}+\varepsilon$ and consider a perturbation $\tilde{\mathrm{y}}(\cdot) \in C^{1}$ of the boundary $\mathrm{y}(\cdot)$ which satisfies the conditions of Theorem 5.6 and $\mathrm{y}(\theta)<\tilde{\mathrm{y}}(\theta) \leq y_{0}$ in $(\tilde{\mathrm{y}}(\theta), \theta) \in U$ and such that $\tilde{\mathrm{y}}$ and y coincide outside of $U$. For the corresponding reflection strategies $\tilde{A}:=A^{\mathrm{refl}}\left(\tilde{\mathrm{y}}, \Theta_{0}\right)$ and $A:=A^{\mathrm{refl}}\left(\mathrm{y}, \Theta_{0}\right)$ denote by $\tilde{\Theta}_{t}:=\Theta_{0}-\tilde{A}_{t}$ and $\Theta_{t}:=\Theta_{0}-A_{t}$ their asset position processes. The liquidation times of $\tilde{A}$ and $A$ are $\tilde{\tau}:=\inf \left\{t \geq 0: \tilde{A}_{t}=\Theta_{0}\right\}$ and $\tau:=\inf \left\{t \geq 0: A_{t}=\Theta_{0}\right\}$, respectively. By Theorem 4.3 (see also the discussion after (3.5)), we have $T:=\tilde{\tau} \vee \tau<\infty$ a.s. Fix initial impact $Y_{0-}^{\tilde{A}}=Y_{0-}^{A}=\mathrm{y}\left(\Theta_{0}\right)$. To compare the strategies $A$ and $\tilde{A}$, consider the processes $G\left(\mathrm{y}\left(\Theta_{0}\right) ; A\right)$ and $G\left(\mathrm{y}\left(\Theta_{0}\right) ; \tilde{A}\right)$ from (3.1) for our candidate value function (which is $C^{2,1}$ by Lemma 6.5). Since $V(\cdot, 0)=0$, we have $L_{T}(\tilde{A})=G_{T}(\tilde{A})$ and $L_{T}(A)=G_{T}(A)$. However, since $\left(Y^{\tilde{A}}, \tilde{\Theta}\right)$ spends a positive amount of time in the region $\{\mathcal{L} V>0\}$ until time $T$ and always remains in the region $\{\mathcal{L} V \geq 0\}$, the perturbed strategy $\widetilde{A}$ generates larger proceeds (in expectation) than $A$.

Indeed, by (3.2) applied for $G(\tilde{A})$ and $G(A)$, using monotone convergence (twice) and arguments as in the proof of Proposition 6.1 for the first equality (by (4.8) expected proceeds are bounded), and Lemma 6.2 for the stochastic integrals in the second line (noting the growth condition from Lemma 6.5), we get

$$
\begin{aligned}
\mathbb{E}\left[L_{\infty}(\tilde{A})\right. & \left.-L_{\infty}(A)\right]=\lim _{n \rightarrow \infty} \mathbb{E}\left[G_{n \wedge T}(\tilde{A})-G_{n \wedge T}(A)\right] \\
& =\lim _{n \rightarrow \infty} \mathbb{E}\left[\int_{0}^{n \wedge T} \ldots \mathrm{d} W_{t}+\int_{0}^{n \wedge T} \ldots \mathrm{d} B_{t}+\int_{0}^{n \wedge T} \mathcal{L} V\left(Y_{t}^{\tilde{A}}, \tilde{\Theta}_{t}\right) \mathrm{d} t\right] \\
& =\mathbb{E}\left[\int_{0}^{T} \mathcal{L} V\left(Y_{t}^{\tilde{A}}, \tilde{\Theta}_{t}\right) \mathrm{d} t\right]>0 .
\end{aligned}
$$

This contradicts Theorem 5.6, so $h^{\prime}(0+) \leq 0$ and hence the inequality in $\mathcal{S}_{1}$ must hold.

It remains to consider the case $(y, \theta) \in \overline{\mathcal{S}}_{2}$, where $V_{y}^{\mathcal{S}_{2}}=f(y)-f(y-\theta)$ and $V_{y y}^{\mathcal{S}_{2}}=f^{\prime}(y)-f^{\prime}(y-\theta)$. Fix $y-\theta=: a \geq y_{0}$ and consider $\mathcal{L} V^{\mathcal{S}_{2}}$ as a function of $\theta$. We have

$$
\begin{aligned}
\mathcal{L} V^{\mathcal{S}_{2}}(y, \theta)=\frac{\hat{\sigma}^{2}}{2} & \left(f^{\prime}(a+\theta)-f^{\prime}(a)\right)+(\sigma \rho \hat{\sigma}-\beta(a+\theta))(f(a+\theta)-f(a)) \\
& -\delta \int_{a}^{a+\theta} f(x) \mathrm{d} x .
\end{aligned}
$$

Differentiating the right-hand side w.r.t. $\theta$ we get $f(a+\theta) k(a+\theta)$, which is again decreasing in $\theta$ because $a \geq y_{0}$. Since at $\theta=0$ we have $\mathcal{L} V^{\mathcal{S}_{2}}(y, \theta)=0$ we deduce the desired inequality.

Remark 6.8. In the particular case when $\lambda=f^{\prime} / f$ is constant, a more direct approach based on straightforward calculations leads to a conjecture on a property for quotients 
of Hermite functions. More precisely, to prove $h^{\prime}(0+) \leq 0$ in this case it turns out to be sufficient to verify that the map $y_{b} \mapsto h^{\prime}(0)$ is monotone in $\left[y_{\infty}, y_{0}\right]$, because at $y_{\infty}$ and $y_{0}$ one can check that $h^{\prime}(0+)<0$. The monotonicity in $y_{b}$ would then follow from the following conjectured property of the Hermite functions:

$$
\text { For every } \nu<0 \text {, the function } \quad x \mapsto \frac{\left(H_{\nu-1}(x)\right)^{2}}{H_{\nu}(x) H_{\nu-2}(x)} \quad \text { is decreasing. }
$$

Numerical computations indicate the validity of the this property but, to our best knowledge, it is not yet proven and may be of independent interest. Note that such quotients of special functions are related to so called Turan-type inequalities, cf. [BI13].

Now we have all the ingredients in place to complete the

Proof of Theorem 3.1. The function $V$ constructed in 6.6 is a classical solution of the variational inequality $(3.3)$ because of Lemmas 6.5, 6.6 and 6.7. Thus, for each admissible strategy $A$ the process $G(y ; A)$ from $(3.1)$ is a supermartingale with $G_{0}(y ; A) \leq G_{0-}(y ; A)$ : the growth condition on $V_{y}$ and $V$ from Lemma 6.5 guarantees that the stochastic integral processes in 3.2 are true martingales by an application of Lemma 6.2, while the variational inequality gives the supermartingale property on $[0-, \infty)$. Moreover, for the described strategy $A^{*}$, whose existence and uniqueness on $\llbracket 0, \tau \rrbracket$ follows from classical results, cf. Remark 4.2 the process $G\left(y ; A^{*}\right)$ is a true martingale with $G_{0}\left(y ; A^{*}\right)=G_{0-}\left(y ; A^{*}\right)$ by our construction of $V$ and the validity of the variational inequality in the respective regions. Therefore $A^{*}$ is an optimal strategy by Proposition 6.1. Any other strategy will be suboptimal because the respective inequalities are strict in the sell and wait region, i.e., for any other strategy the process $G$ will be a strict supermartingale.

The Laplace transform formula (3.5) was derived in Theorem 4.3 for a $\mathbf{y}$-reflected strategy when the state process starts on the boundary. If the state process starts in $Y_{0}=x$ in the wait region, the behavior of the process until time $H^{x \rightarrow z}$ when it hits the boundary for the first time (at $z:=\mathbb{y}\left(\Theta_{0}\right)$ ) is independent from future excursions from the boundary, and hence the multiplicative factor in (3.5), see e.g. [RW87, Prop. V.50.3]: for $x<z \in \mathbb{R}$ and $\alpha>0, \mathbb{E}\left[\exp \left(-\alpha H^{x \rightarrow z}\right)\right]=\Phi_{\alpha}(x) / \Phi_{\alpha}(z)$.

\section{References}

[AFS10] Aurélien Alfonsi, Antje Fruth, and Alexander Schied. Optimal execution strategies in limit order books with general shape functions. Quant. Finance, 10(2):143-157, 2010.

[AKS16] Aurélien Alfonsi, Florian Klöck, and Alexander Schied. Multivariate transient price impact and matrix-valued positive definite functions. Math. Oper. Res., 41(3):914-934, 2016.

[Alm12] Robert Almgren. Optimal trading with stochastic liquidity and volatility. SIAM J. Financial Math., 3(1):163-181, 2012. 
[AW95] Joseph Abate and Ward Whitt. Numerical inversion of Laplace transforms of probability distributions. ORSA J. Comput., 7(1):36-43, 1995.

[BBF17a] Dirk Becherer, Todor Bilarev, and Peter Frentrup. Optimal asset liquidation with multiplicative transient price impact. Appl. Math. Optim., 2017.

[BBF17b] Dirk Becherer, Todor Bilarev, and Peter Frentrup. Stability for gains from large investors' strategies in M1/J1 topologies. 2017. arXiv:1701.02167v1.

[BI13] Árpád Baricz and Mourad E. H. Ismail. Turán type inequalities for Tricomi confluent hypergeometric functions. Constr. Approx., 37(2):195-221, 2013.

[CL95] Louis K. C. Chan and Josef Lakonishok. The behavior of stock prices around institutional trades. J. Finance, 50(4):1147-1174, 1995.

[DI93] Paul Dupuis and Hitoshi Ishii. SDEs with oblique reflection on nonsmooth domains. Ann. Probab., 21(1):554-580, 1993.

[DM82] Claude Dellacherie and Paul-André Meyer. Probabilities and Potential B: Theory of Martingales. North-Holland, Amsterdam, 1982.

[Fre98] Rüdiger Frey. Perfect option hedging for a large trader. Finance Stoch., 2(2):115-141, 1998.

[FSU17] Antje Fruth, Torsten Schöneborn, and Mikhail Urusov. Optimal trade execution in order books with stochastic liquidity. Preprint, available at http://homepage. alice. de/murusov/papers/fsu-optimal_ execution_stochastic. pdf, 2017.

[GF00] Israel M. Gelfand and Sergei V. Fomin. Calculus of Variations. Dover Books on Mathematics. Dover Publications, 2000.

[GH16] Paulwin Graewe and Ulrich Horst. Optimal trade execution with instantaneous price impact and stochastic resilience. SIAM J. Control Optim., 2016. to app.

[GHS16] Paulwin Graewe, Ulrich Horst, and Eric Séré. Smooth solutions to portfolio liquidation problems under price-sensitive market impact. Stoch. Process. Appl., 2016.

[JS03] Jean Jacod and Albert N. Shiryaev. Limit Theorems for Stochastic Processes. Springer, Berlin, second edition, 2003.

[Kle08] Achim Klenke. Probability Theory: A Comprehensive Course. Universitext. Springer London, 2008.

[KS86] Ioannis Karatzas and Steven E. Shreve. Equivalent models for finite-fuel stochastic control. Stochastics, 18(3-4):245-276, 1986. 
[Kyl85] Albert S. Kyle. Continuous auctions and insider trading. Econometrica, 53(6):1315-1335, 1985.

[Leb72] Nikolaŭ N. Lebedev. Special Functions and Their Applications. Dover Books on Mathematics. Dover Publications, 1972.

[LN17] Charles-Albert Lehalle and Eyal Neuman. Incorporating signals into optimal trading. 2017. arXiv:1704.00847v2.

[LS13] Christopher Lorenz and Alexander Schied. Drift dependence of optimal trade execution strategies under transient price impact. Finance Stoch., 17(4):743-770, 2013.

[PSS11] Silviu Predoiu, Gennady Shaikhet, and Steven Shreve. Optimal execution in a general one-sided limit-order book. SIAM J. Financial Math., 2(1):183-212, 2011.

[PTW07] Guodong Pang, Rishi Talreja, and Ward Whitt. Martingale proofs of manyserver heavy-traffic limits for Markovian queues. Probab. Surveys, 4:193-267, 2007.

[RW87] L. Chris G. Rogers and David Williams. Diffusions, Markov Processes and Martingales, Vol. II: Itô Calculus. Cambridge University Press, second edition, 1987.

[SZ17] Alexander Schied and Tao Zhang. A state-constrained differential game arising in optimal portfolio liquidation. Math. Finance, 27(3):779-802, 2017. 